\title{
Cortical rich club regions can organize state- dependent functional network formation by engaging in oscillatory behavior
}

Citation for published version (APA):

Senden, M., Reuter, N., van den Heuvel, M. P., Goebel, R., \& Deco, G. (2017). Cortical rich club regions can organize state-dependent functional network formation by engaging in oscillatory behavior. Neuroimage, 146, 561-574. https://doi.org/10.1016/j.neuroimage.2016.10.044

Document status and date:

Published: 01/02/2017

DOI:

10.1016/j.neuroimage.2016.10.044

Document Version:

Publisher's PDF, also known as Version of record

Document license:

Taverne

Please check the document version of this publication:

- A submitted manuscript is the version of the article upon submission and before peer-review. There can be important differences between the submitted version and the official published version of record.

People interested in the research are advised to contact the author for the final version of the publication, or visit the DOI to the publisher's website.

- The final author version and the galley proof are versions of the publication after peer review.

- The final published version features the final layout of the paper including the volume, issue and page numbers.

Link to publication

\footnotetext{
General rights rights.

- You may freely distribute the URL identifying the publication in the public portal. please follow below link for the End User Agreement:

www.umlib.nl/taverne-license

Take down policy

If you believe that this document breaches copyright please contact us at:

repository@maastrichtuniversity.nl

providing details and we will investigate your claim.
}

Copyright and moral rights for the publications made accessible in the public portal are retained by the authors and/or other copyright owners and it is a condition of accessing publications that users recognise and abide by the legal requirements associated with these

- Users may download and print one copy of any publication from the public portal for the purpose of private study or research.

- You may not further distribute the material or use it for any profit-making activity or commercial gain

If the publication is distributed under the terms of Article $25 \mathrm{fa}$ of the Dutch Copyright Act, indicated by the "Taverne" license above, 


\title{
Cortical rich club regions can organize state-dependent functional network formation by engaging in oscillatory behavior
}

\author{
Mario Senden $^{\mathrm{a}, \mathrm{b}, *}$, Niels Reuter ${ }^{\mathrm{a}, \mathrm{g}}$, Martijn P. van den Heuvel ${ }^{\mathrm{d}}$, Rainer Goebel ${ }^{\mathrm{a}, \mathrm{b}, \mathrm{c}, 1}$, \\ Gustavo Deco ${ }^{\mathrm{e}, \mathrm{f}, 1}$ \\ a Department of Cognitive Neuroscience, Faculty of Psychology and Neuroscience, Maastricht University, 6201BC Maastricht, The Netherlands \\ b Maastricht Brain Imaging Centre, Faculty of Psychology and Neuroscience, Maastricht University, P.O. Box 616, 6200 MD Maastricht, The Netherlands \\ ${ }^{\mathrm{c}}$ Department of Neuroimaging and Neuromodeling, Netherlands Institute for Neuroscience, an Institute of the Royal Netherlands Academy of Arts and \\ Sciences (KNAW), 1105BA Amsterdam, The Netherlands \\ d Brain Center Rudolf Magnus, Department of Psychiatry, University Medical Center Utrecht, Brain Center Rudolf Magnus, 3508 GA Utrecht, The \\ Netherlands \\ e Center for Brain and Cognition, Computational Neuroscience Group, Department of Information and Communication Technologies, Universitat Pompeu \\ Fabra, Roc Boronat 138, Barcelona 08018, Spain \\ ${ }^{\mathrm{f}}$ Institució Catalana de la Recerca i Estudis Avançats (ICREA), Universitat Pompeu Fabra, Passeig Lluís Companys 23, Barcelona 08010, Spain \\ ${ }^{\mathrm{g}}$ Institute of Neuroscience and Medicine (INM-1) Research Centre Jülich
}

\begin{abstract}
A B S T R A C T
Cognition is hypothesized to require the globally coordinated, functionally relevant integration of otherwise segregated information processing carried out by specialized brain regions. Studies of the macroscopic connectome as well as recent neuroimaging and neuromodeling research have suggested a densely connected collective of cortical hubs, termed the rich club, to provide a central workspace for such integration. In order for rich club regions to fulfill this role they must dispose of a dynamic mechanism by which they can actively shape networks of brain regions whose information processing needs to be integrated. A potential candidate for such a mechanism comes in the form of oscillations which might be employed to establish communication channels among relevant brain regions. We explore this possibility using an integrative approach combining whole-brain computational modeling with neuroimaging, wherein we investigate the local dynamics model brain regions need to exhibit in order to fit (dynamic) network behavior empirically observed for resting as well as a range of task states. We find that rich club regions largely exhibit oscillations during task performance but not during rest. Furthermore, oscillations exhibited by rich club regions can harmonize a set of asynchronous brain regions thus supporting functional coupling among them. These findings are in line with the hypothesis that the rich club can actively shape integration using oscillations.
\end{abstract}

\section{Introduction}

The human brain is characterized by a high degree of structural segregation allowing for designated information processing within specialized brain regions (Bear et al., 2006; Flourens, 1842; Lashley, 1929). While this is beneficial for unimodal and automatic processing, higher cognition is hypothesized to require the globally coordinated integration of segregated brain regions into temporal functional networks (Baars, 2005; Deco et al., 2011; Dehaene and Naccache, 2001; Ghosh et al., 2008). A high degree of integration is thus additionally required for efficient information processing (Damasio, 1989; Tononi,
2004). Studies of human, macaque, and other mammal cortices have shown the presence of a hierarchically higher module termed the rich club which is characterized by hubs with dense intra- and intermodular connectivity (Colizza et al., 2006; van den Heuvel and Sporns, 2011; Zamora-López et al., 2009). The rich club forms a structural backbone mediating a majority of all anatomical paths between pairs of brain regions (van den Heuvel et al., 2012; ZamoraLópez et al., 2009), suggesting a prominent role for it in functional integration.

In accordance with this, recent functional magnetic resonance imaging (fMRI) studies have shown that cortical hubs carry traces of

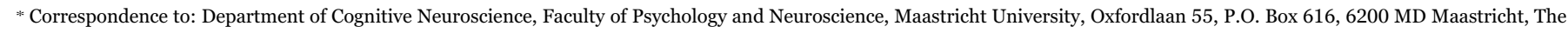
Netherlands.

E-mail address: mario.senden@maastrichtuniversity.nl (M. Senden).

${ }^{1}$ These authors contributed equally to the paper. 
blood oxygen-level dependent (BOLD) activity of resting- and task related functional networks (Braga et al., 2013; Leech et al., 2012), indicative of communication among brain regions being mediated by rich club regions. fMRI research has further shown that cortical hubs update their pattern of global functional connectivity in response to changing task demands (Cole et al., 2013). Additionally, simulation studies employing a steady-state attractor model have suggested that cortical hubs, and specifically the rich club, may allow the brain to sustain a large functional repertoire characterized by diverse configurations of peripheral, i.e. low degree, regions around a stable highdegree core (Deco et al., 2012; Senden et al., 2014). Similar observations have been made in studies using oscillatory neural mass models showing that the rich club can facilitate the synchronization among groups of cortical regions (Gollo et al., 2015; Schmidt et al., 2015). The latter is especially interesting given that cortical regions can undergo transitions from asynchronous to oscillatory behavior as a result of interactions between cortical and thalamic neuronal populations in the presence of noise (Breakspear et al., 2006).

These findings suggest that rich club regions may utilize oscillations as a local control mechanism to organize brain regions into functional networks as they dynamically adjust their oscillatory behavior in response to changing task demands. Furthermore, if rich club regions are generally involved in functional integration, these adjustments should occur irrespective of cognitive domain. The aim of the present study is to investigate this possibility by combining fMRI measurements of resting and task states with simulations of a whole-brain model whose cortical regions can exhibit local dynamics ranging from asynchronous fluctuations (noise diffusion) to structured oscillations. We restrict our investigations to oscillations falling within the infraslow frequency band (defined as the range from $.01 \mathrm{~Hz}$ to $.2 \mathrm{~Hz}$; Vanhatalo et al., 2004) due to the slow nature of the BOLD signal. These oscillations might be a proxy for low-pass filtered oscillatory behavior at higher frequency bands. However, prior research suggests that the infraslow frequency band might also be relevant for functional network formation in its own right since oscillatory behavior in this band has been shown to modulate regions' excitability states and has been implicated in resting state and task execution (Hiltunen et al., 2014; Monto et al., 2008; Vanhatalo et al., 2004). To study the relevance of local oscillatory behavior for whole-brain dynamics related to cognition irrespective of a specific cognitive domain, we obtained fMRI data for a range of tasks, including an n-Back task (Kirchner, 1958), the Eriksen Flanker task (Eriksen and Eriksen, 1974), a mental rotation task (Shepard and Metzler, 1971), and a verbal odd-man-out task (Flowers and Robertson, 1985). These tasks were chosen to tap into working memory, executive function and inhibition, mental rotation, and semantic reasoning, respectively. These cognitive domains are conceptually different and their associated functional connectivity profiles have been shown to be minimally overlapping (Smith et al., 2009). The combined simulation and fMRI approach allows us to investigate the local dynamics of rich club regions as opposed to other cortical regions, whether rich club regions adapt their local dynamics in response to task performance as compared to rest, and how this relates to whole-brain functional coupling.

\section{Materials and methods}

\section{Participants}

Fourteen healthy subjects ( 8 females, age range $=22-43$ years, mean age $=28.76$ years) were recruited for resting state and task related fMRI measurements. All subjects had normal or corrected-to-normal visual acuity, were screened, and provided written informed consent prior to scanning.

\section{Task and stimulus description}

In the present study subjects underwent six functional runs. These runs consisted of a resting-state measurement, four individual task measurements including an n-Back $(n=2)$ task (Kirchner, 1958), the Eriksen Flanker task (Eriksen and Eriksen, 1974), a mental rotation task (Shepard and Metzler, 1971), an odd-man-out task (Flowers and Robertson, 1985), and a task-switching paradigm wherein participants repeatedly performed each of the four tasks. Using the cognitive atlas (http://www.cognitiveatlas.org; Poldrack et al., 2011), tasks were selected based on how well they reflect a specific cognitive domain. The cognitive domains (working memory, executive function \& inhibition, mental rotation, and semantic reasoning), in turn, were chosen because they show distinctive patterns of mapping to the ten primary resting-state functional networks (Smith et al., 2009). All resting-state measurements preceded task-related measurements to prevent carry-over effects (Grigg and Grady, 2010). Resting-state runs lasted for 8 minutes during which subjects were instructed to close their eyes. The four individual task runs followed rest, lasted $\sim 7 \mathrm{~min}$ each, and were counter-balanced across participants. The taskswitching run lasted $9 \mathrm{~min}$ and was always performed last to allow participants to get familiar with performing each task in the scanner before being required to switch between them. Since this run was not the object of the present study it will not be discussed further. Descriptions of the remaining four tasks are as follows:

\section{N-back Task}

In a visual 2-back task subjects were presented with a sequence of abstract shape stimuli and instructed to indicate whether the currently presented stimulus matches the second to last stimulus in the sequence presented before. Abstract, snowflake-like, shapes were used in order to prevent subjects from translating a visual into a verbal representation and thus minimizing representational overlap with the verbal oddman-out task. A total of 9 different snowflake-like shapes were shown in a quasi-random order. Shape presentation trials lasted for $1 \mathrm{~s}$ followed by a $1 \mathrm{~s}$ inter-trial interval. In total there were 192 trials forming a single task block preceded and followed by a $16 \mathrm{~s}$ rest period leading to a total run length of $416 \mathrm{~s}$. Of the 192 trials 48 (25\%) required a response indicating a match.

\section{Flanker Task}

In a modified version of the Eriksen Flanker task (Eriksen and Eriksen, 1974) subjects were presented with three rows of arrow stimuli pointing either to the right or the left. The center arrow was the target stimulus indicating whether a left or right response was required. The arrows surrounding the target stimulus were distractors whose direction was either congruent or incongruent with that of the target stimulus. Trials lasted for $1 \mathrm{~s}$ followed by a $1 \mathrm{~s}$ inter-trial interval. In total there were 192 trials forming a single task block preceded and followed by a $16 \mathrm{~s}$ rest period leading to a total run length of $416 \mathrm{~s}$. Of the 192 trials 96 (50\%) were incongruent thus requiring response inhibition.

\section{D Mental rotation task}

In a mental rotation task subjects were required to mentally rotate a three-dimensional probe stimulus in order to confirm or deny an identity match with a target stimulus. Subjects could indicate a match between the two by pressing the 'right' button and a mismatch by pressing the 'left' button. Trials lasted for $3 \mathrm{~s}$ followed by a $1 \mathrm{~s}$ intertrial interval. In total there were 96 trials forming a single task block preceded and followed by a $16 \mathrm{~s}$ rest period leading to a total run length of 416 s. Of the 96 trials 24 (25\%) presented matching probe and target stimuli.

\section{Odd-Man-Out task}

In a verbal odd-man-out task subjects were presented with three 
words and had to indicate which of the three semantically fits least with the others. The word being the odd-man-out in a specific trial was always either presented on the outer left or the outer right with the middle word forming a semantic anchor. Hence, the required response was a 'left' or 'right' button press, if the odd-man-out was presented on the left or the right of the anchor, respectively. Trials lasted for $3 \mathrm{~s}$ followed by a $1 \mathrm{~s}$ inter-trial interval. In total there were 96 trials forming a single task block preceded and followed by a $16 \mathrm{~s}$ rest period leading to a total run length of $416 \mathrm{~s}$.

\section{Stimulus presentation}

All tasks were programmed in Presentation ${ }^{\circledR}$ (Version 10; www. neurobs.com). Stimuli were presented to the subjects at a resolution of $1920 \times 1200$ pixels. All experiments were performed on a hardware configuration containing a Dell Optiplex 970 computer with a NVIDIA NVS 300 graphics card with OpenGL $>2.0$ support connected to a Panasonic PT EZ570E wuxga projector. The projected stimuli were first reflected off a mirror positioned behind the bore of the magnet before they were reflected off a second mirror located above the head coil. The projection pathway together with the scanner table positioning created a fixed maximum visual angle of $18.76^{\circ}$ for all subjects.

\section{Magnetic resonance imaging}

Images were acquired at Maastricht Brain Imaging Centre (Maastricht University) on a $3 \mathrm{~T}$ scanner (Tim Trio/upgraded to Prisma Fit, Siemens Healthcare, Germany). Anatomical data were collected prior to functional data with a T1-weighted MPRAGE imaging sequence (192 sagittal slices; Repetition Time [TR]=2250 ms; Echo Time $[\mathrm{TE}]=2.21 \mathrm{~ms}$; Flip Angle $[\mathrm{FA}]=9^{\circ}$; Field of View $[\mathrm{FoV}]$ $=256 \times 256 \mathrm{~mm}^{2} ; 1 \mathrm{~mm}$ isotropic resolution. Functional images were acquired using a gradient-echo echo-planar imaging sequence (38 transversal slices; $\quad \mathrm{TR}=2000 \mathrm{~ms} ; \quad \mathrm{TE}=30 \mathrm{~ms} ; \quad \mathrm{FA}=77^{\circ}$; FoV $=216 \times 216 \mathrm{~mm}^{2} ; \quad$ voxel-size $=3 \times 3 \times 3.5 \mathrm{~mm}^{3} ; \quad$ no slice gap; GRAPPA=3).

\section{Processing of (f)MRI data}

Anatomical images were automatically processed with the longitudinal stream in FreeSurfer (Reuter et al., 2012; http://surfer.nmr. mgh.harvard.edu/) including probabilistic atlas based cortical parcellation according to the Desikan-Killany (DK) atlas (Desikan et al., 2006). Initial preprocessing of each functional dataset was performed in BrainVoyager QX (v2.6; Brain Innovation, Maastricht, the Netherlands) and included slice scan time correction, (rigid body) motion correction, and high-pass filtering with a frequency cutoff of $.01 \mathrm{~Hz}$. Due to the use of preparation scans, none of the initial volumes needed to be discarded related to T1 equilibrium effects. Subsequently, functional images were registered to the subject's anatomical images. Using MATLAB (2013a, The MathWorks,Natick, MA), signals were then cleaned further by performing wavelet despiking (Patel and Bullmore, 2015) and regressing out a global noise signal given by the first principal component of signals observed within the cerebrospinal fluid of the ventricles. Next, voxels were uniquely assigned to one of 68 cortical regions and an average BOLD signal for each region was computed as the mean time-series over all voxels of that region. Finally, since the initial and final 8 data points of all task runs constitute a rest-period, these data points were removed leaving 192 data points during which a task was continuously performed. For reasons of comparability, resting state BOLD signal was equally reduced to 192 data points. However, all analyses and simulations were repeated with the full resting state data set and did not produce different results.
Table 1

Cortical regions (ordered).

\begin{tabular}{|c|c|}
\hline$\#$ & Region Name \\
\hline 1 & left banks of the superior temporal sulcus \\
\hline 2 & left caudalanterior-cingulate cortex \\
\hline 3 & left caudalmiddle frontal gyrus \\
\hline 4 & left cuneus \\
\hline 5 & left entorhinal cortex \\
\hline 6 & left fusiform gyrus \\
\hline 7 & left inferior parietal cortex \\
\hline 8 & left inferior temporal cortex \\
\hline 9 & left isthmus-cingulate cortex \\
\hline 10 & left lateral occipitalcortex \\
\hline 11 & left lateral orbital frontal cortex \\
\hline 12 & left lingual gyrus \\
\hline 13 & left medial orbital frontal cortex \\
\hline 14 & left middle temporal gyrus \\
\hline 15 & left parahippocampal gyrus \\
\hline 16 & left paracentral lobule \\
\hline 17 & left pars opercularis \\
\hline 18 & left pars orbitalis \\
\hline 19 & left pars triangularis \\
\hline 20 & left pericalcarine cortex \\
\hline 21 & left postcentral gyrus \\
\hline 22 & left posterior-cingulate cortex \\
\hline 23 & left precentral gyrus \\
\hline 24 & left rostralanterior cingulate cortex \\
\hline 25 & left rostralmiddle frontal gyrus \\
\hline 26 & left superior temporal gyrus \\
\hline 27 & left supramarginal gyrus \\
\hline 28 & left frontal pole \\
\hline 29 & left temporal pole \\
\hline 30 & left transverse temporal cortex \\
\hline 31 & left insula \\
\hline 32 & left precuneus \\
\hline 33 & left superior frontal cortex \\
\hline 34 & left superior parietal cortex \\
\hline 35 & right superior parietal cortex \\
\hline 36 & right superior frontal cortex \\
\hline 37 & right precuneus \\
\hline 38 & right insula \\
\hline 39 & right transverse temporal cortex \\
\hline 40 & right temporal pole \\
\hline 41 & right frontal pole \\
\hline 42 & right supramarginal gyrus \\
\hline 43 & right superior temporal gyrus \\
\hline 44 & right rostralmiddle frontal gyrus \\
\hline 45 & right rostralanterior cingulate cortex \\
\hline 46 & right precentral gyrus \\
\hline 47 & right posterior-cingulate cortex \\
\hline 48 & right postcentral gyrus \\
\hline 49 & right pericalcarine cortex \\
\hline 50 & right pars triangularis \\
\hline 51 & right pars orbitalis \\
\hline 52 & right pars opercularis \\
\hline 53 & right paracentral lobule \\
\hline 54 & right parahippocampal gyrus \\
\hline 55 & right middle temporal gyrus \\
\hline 56 & right medial orbital frontal cortex \\
\hline 57 & right lingual gyrus \\
\hline 58 & right lateral orbital frontal cortex \\
\hline 59 & right lateral occipitalcortex \\
\hline 60 & right isthmus-cingulate cortex \\
\hline 61 & right inferior temporal cortex \\
\hline 62 & right inferior parietal cortex \\
\hline 63 & right fusiform gyrus \\
\hline 64 & right entorhinal cortex \\
\hline 65 & right cuneus \\
\hline 66 & right caudalmiddle frontal gyrus \\
\hline 67 & right caudalanterior-cingulate cortex \\
\hline 68 & right banks of the superior temporal sulcus \\
\hline
\end{tabular}

\section{Structural connectivity}

High-quality diffusion-weighted MRI data of 215 subjects was obtained from the human connectome project's (HCP) Q3 release 
A)

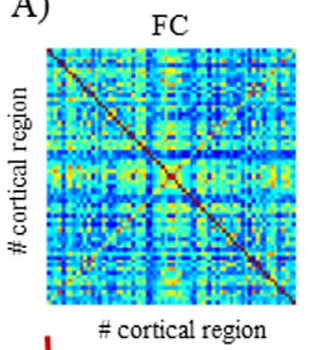

$\mathrm{DFC}$

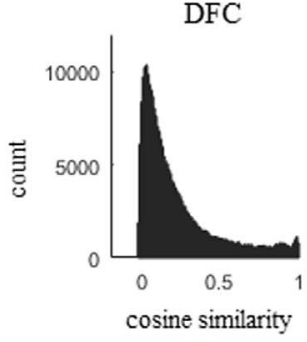

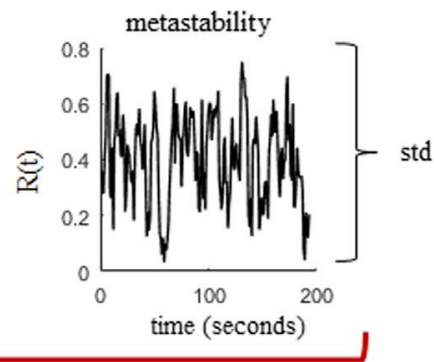

$$
\begin{aligned}
& \frac{d x_{j}}{d t}=\left[\left(A-x_{j}^{2}-y_{j}^{2}\right] x_{j}-\omega_{j} y_{j}+G \sum_{i} C_{i j}\left(x_{i}-x_{j}\right)+\beta \eta_{j}(t)\right. \\
& \frac{d y_{j}}{d t}=\left[A-x_{j}^{2}-y_{j}^{2}\right] y_{j}+\omega_{j} x_{j}+G \sum_{i} C_{i j}\left(y_{i}-y_{j}\right)+\beta \eta_{j}(t)
\end{aligned}
$$

B)

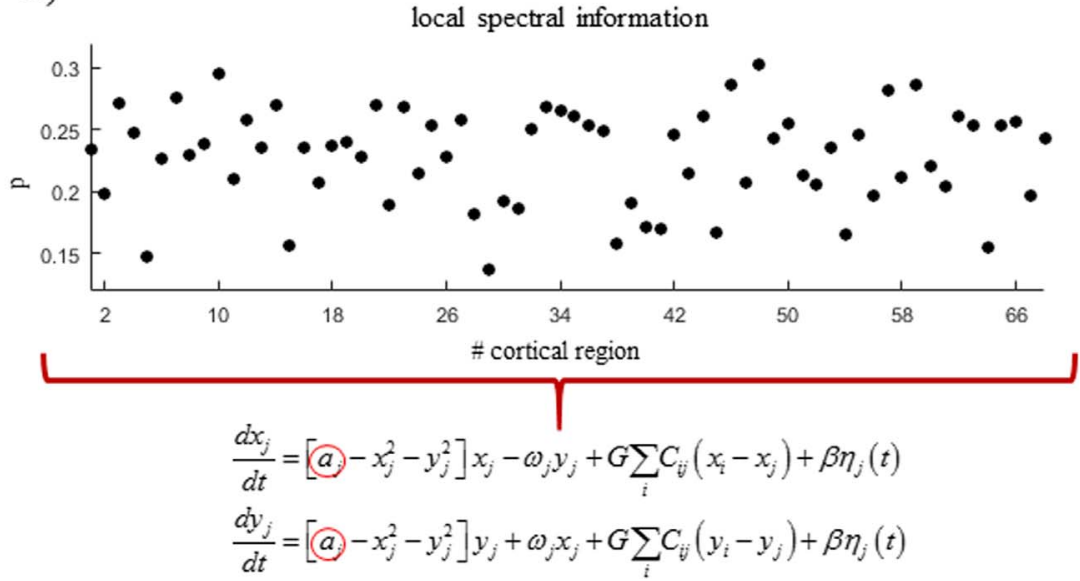

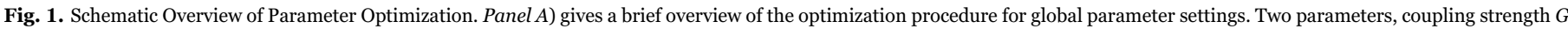

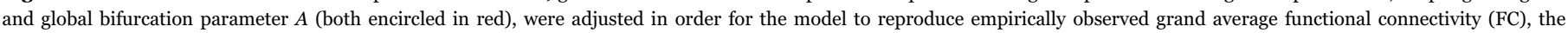

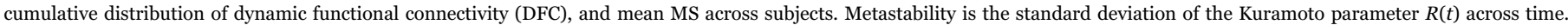

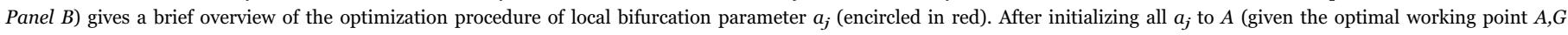

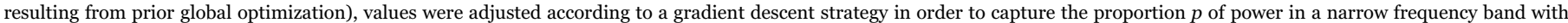

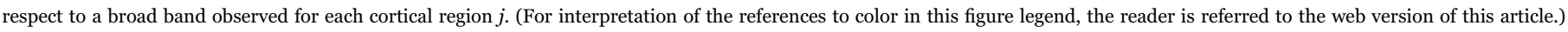

(Glasser et al., 2013; Van Essen et al., 2012). White matter fibers were traced for each subject using generalized q-sampling imaging (GQI) and streamline tractography (Yeh et al., 2010) and the cortex was parcellated into 68 cortical regions based on the DK atlas (Desikan et al., 2006). More details on these processing steps can be found elsewhere (de Reus and van den Heuvel, 2014). A weighted structural connectivity matrix was then generated by averaging streamlines over subjects, keeping only those entries which had positive values for at least $60 \%$ of subjects (de Reus and van den Heuvel, 2013), and resampling the data to follow a Gaussian distribution with a mean of .5 and a standard deviation of .15 (Honey et al., 2009). Gaussian resampling of, or alternatively log transforming, the data has recently been shown to enhance correspondence between diffusion tractography and in vivo animal tract-tracing measurements of anatomical connectivity (van den Heuvel et al., 2015) (Table 1).

\section{Whole-brain model}

To examine the interplay of local and global dynamics during rest as well as in response to task demands we studied the behavior of interconnected regions represented by a neural mass model characterized by the normal form of a supercritical Hopf bifurcation and connected according to an anatomical structural connectivity matrix. We chose this description because it can capture transitions from asynchronous to oscillatory behavior. Neural mass models characterized by the normal form of a Hopf bifurcation had previously been shown to provide a good characterization of regional dynamics (Freyer et al., 2011, 2012). Briefly, the dynamic behavior of each region $j$ was given by

$\frac{d z_{j}}{d t}=z_{j}\left[a_{j}+i \omega_{j}-\left|z_{j}\right|^{2}\right]+\beta \eta_{j}(t)$

with $z_{j}$ being complex and hence separable into a real $\left(x_{j}\right)$ and imaginary $\left(y_{j}\right)$ part

$z_{j}=r_{j} e^{i \theta_{j}}=x_{j}+i y_{j}$

and $\eta$ being Gaussian white noise implemented as a Wiener process scaled by a factor $\beta=.02$. In Eq. (1) the bifurcation parameter $a_{j}$ represents a local control variable which determined whether a region was primarily dominated by noisy fluctuations $\left(a_{j}<0\right)$ or by a stable limit cycle with frequency $f_{j}=\omega_{j} / 2 \pi\left(a_{j}>0\right)$. The bifurcation parameter is thus meaningfully interpretable as it gives an indication as to whether brain regions exhibit oscillatory behavior. Supplementary Fig 1 gives a detailed account of a single region thusly described. We also provide code for simulating the interaction among two mutually coupled regions online (https://github.com/MSenden/Hopf.git). Embedding these local dynamics into a large-scale model and separating real from imaginary parts of $z$ leads to whole-brain dynamics defined by the following set of coupled equations:

$\frac{d x_{j}}{d t}=\left[a_{j}-x_{j}^{2}-y_{j}^{2}\right] x_{j}-\omega_{j} y_{j}+G \sum_{i} C_{i j}\left(x_{i}-x_{j}\right)+\beta \eta_{j}(t)$ 
A)

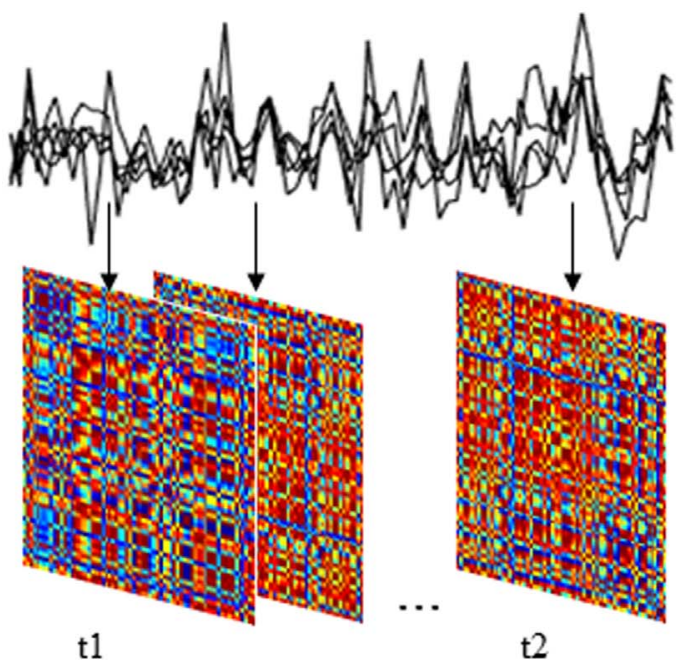

B)

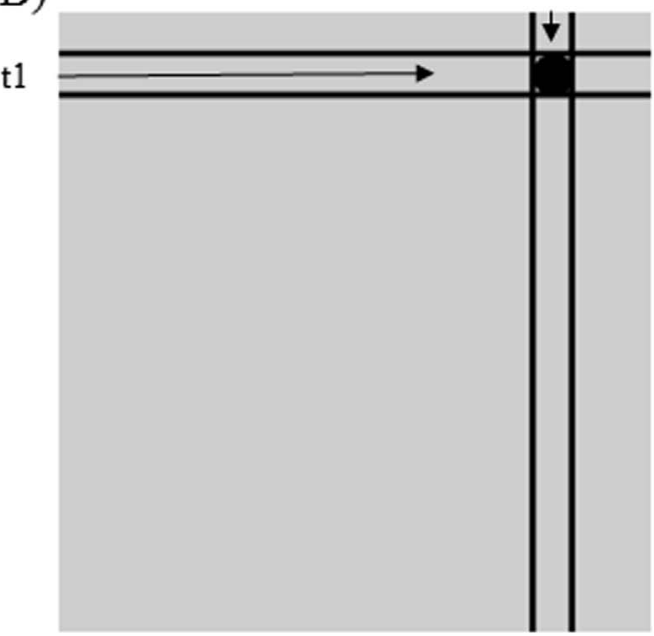

C)

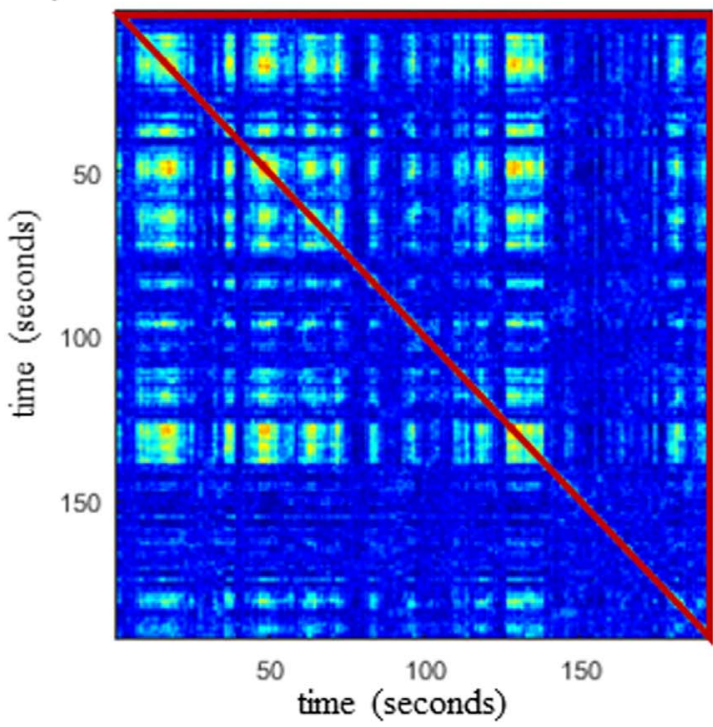

D)

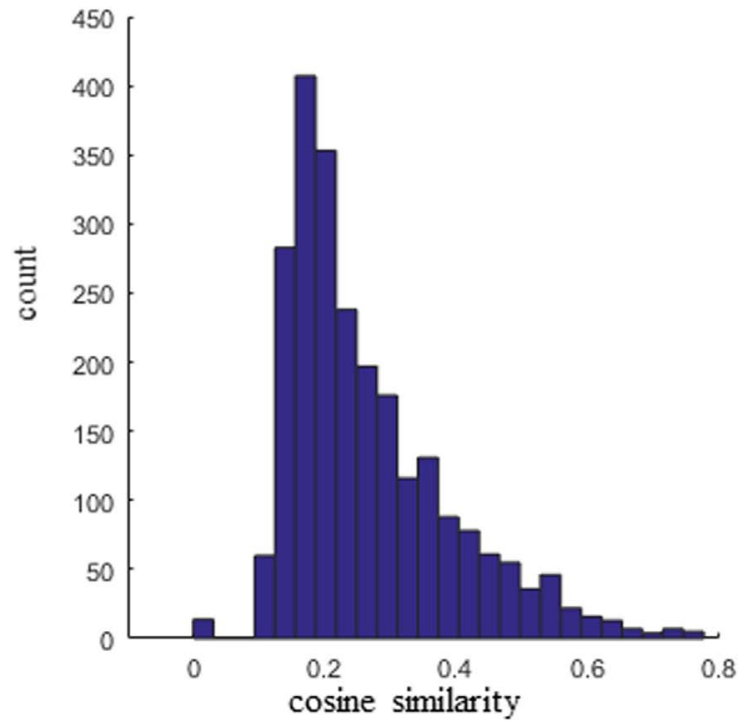

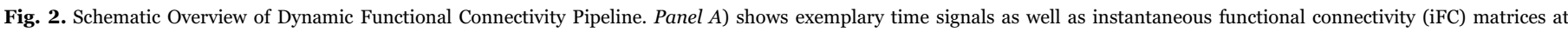

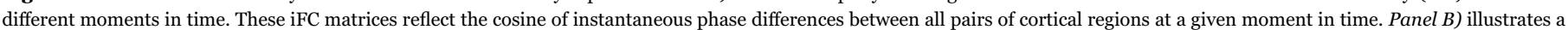

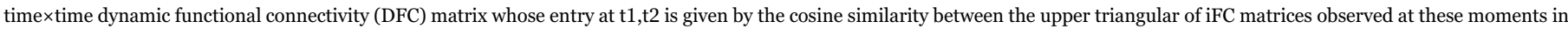

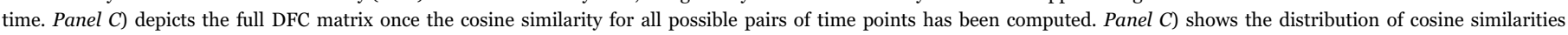

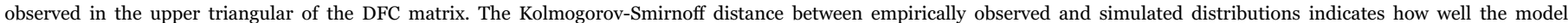
reproduces empirical DFC.

$\frac{d y_{j}}{d t}=\left[a_{j}-x_{j}^{2}-y_{j}^{2}\right] y_{j}+\omega_{j} x_{j}+G \sum_{i} C_{i j}\left(y_{i}-y_{j}\right)+\beta \eta_{j}(t)$

In Eqs. (3) and (4), the coupling strength $G$ scaled the adjacency matrix $C$. Coupling strength is a global control variable separating global dynamics into a unistable regime in the form of a low activity ground state and a multistable regime. Optimal coupling generally occurs close to a bifurcation separating these regimes (Deco et al., 2011; Deco and Jirsa, 2012). Simulated BOLD signal of each area $j$ was directly given by the real part of $z$ (i.e. by $x$ ) with each region having its own characteristic frequency $\omega_{j}$ in the range from $.04 \mathrm{~Hz}$ to $.07 \mathrm{~Hz}$ (a subset of the infraslow frequency range) given by the averaged peak frequency of the empirical BOLD signals in that range (for a distribution of characteristic frequencies per task see supplementary Fig 2). We chose this frequency range as it had previously been identified as reliably reflecting gray matter signals and being minimally affected by aliased physiological noise (Glerean et al., 2012).

\section{Optimization of global control variables}

In order to find the working point for which the model reproduces global dynamics exhibited by the cortex during rest, as well as in response to task demands, two parameters (control variables) needed to be optimized. The first was the previously mentioned global coupling strength $G$ scaling the structural connectivity. The second global parameter was the bifurcation parameter $A$, a fixed value substituted for all local bifurcation parameters $a_{j}$. We characterized global cortex dynamics in terms of grand average functional connectivity (FC), dynamic functional connectivity (DFC), and metastability (MS). Optimization of global control variables involved finding those values of $G$ and $A$ for which the model faithfully reproduced all three metrics as observed in our dataset (see Fig. 1a for a schematic overview).

Grand average functional connectivity

This metric reflects the static component of the relationships 
between region-specific activation patterns. A grand average FC matrix per task (and rest) was obtained by calculating individual FC matrices in the form of pairwise Pearson correlation coefficients of bandpassfiltered (in the range from $.04 \mathrm{~Hz}$ to $.07 \mathrm{~Hz}$ ) regional BOLD signals for each subject, and subsequently averaging across individual subject's FCs. The same general logic applied to the grand average FC obtained for the simulated BOLD signal with the difference that rather than simulating separate subjects, simulation time corresponded to acquisition time multiplied by the number of subjects. The fit between empirical and simulated grand average $\mathrm{FC}$ was considered to be the Pearson correlation coefficient between the entries falling in the upper triangular of the respective matrices. Alternatively, model fit might be considered as the average of Pearson correlations between individual subject's FC and simulated grand average FC. The latter is worth noting since averaging over individual subjects' FCs can affect network characteristics (Moussa et al., 2012). However, both approaches lead to identical fit distributions across parameter space and we only report results from using the grand average FC.

\section{Dynamic functional connectivity}

This metric reflects the dynamics of functional couplings as shortlived global network states dissolve and may re-emerge at different moments in time (Hutchison et al., 2013). The DFC for each task (and rest) was given by the cumulative distribution of the pairwise similarity between instantaneous functional networks across subjects. Specifically, in an individual subject we first computed the analytic signal of each cortical region by applying the Hilbert transformation to its bandpass-filtered BOLD signal. This allowed for the calculation of instantaneous phases (i.e. phases observed at each moment in time) of cortical regions. Next, a phase difference matrix was obtained at each point in time by calculating the instantaneous phase differences between all pairs of cortical regions. These difference matrices were then transformed to similarity matrices by computing the cosine of their entries (Fig. 2a). A single phase similarity matrix reflects the functional connectivity among cortical regions observed at a single moment in time; i.e. the instantaneous functional connectivity (iFC). To estimate the similarity between functional connectivity observed at different moments in time, we calculated the cosine similarity of the upper triangular of iFC matrices between all pairs of time points (Fig. 2b). This results in a DFC matrix (Fig. 2c). The entries falling in the upper triangular of this matrix form the distribution of similarity among pairs of time points in terms of the functional connectivity observed at these moments in time (Fig. 2d). This procedure was repeated for all subjects with the final distribution of cosine similarity values being the aggregated distributions observed for individual subjects. We followed the same logic when calculating the DFC for simulated data. We calculated the Kolmogorov-Smirnoff distance between empirical and simulated distributions in order to evaluate their agreement.

\section{Metastability}

The final metric used here reflects the overall variability of network states of the system; i.e. in how far the system exhibits transient synchronization dynamics (Wildie and Shanahan, 2012). While this metric cannot reveal details with regard to the number or shape of distinct synchronization states, it has previously been shown to be a good constraint for models whose nodes exhibit oscillatory behavior (Cabral et al., 2011; Cabral et al., 2014; Váša et al., 2015). Metastability in each task (and rest) was measured as the standard deviation of the Kuramoto order parameter observed over time. The Kuramoto order parameter $R(t)$ reflects the extent of synchronization exhibited among brain regions at a specific moment in time and is given by

$R(t)=\left|\sum_{j=1}^{n} e^{i \varphi_{j}(t)}\right| / n$ with $\varphi_{j}(t)$ being the instantaneous phase of each bandpass-filtered BOLD signal of regions $j$ at time $t$ and $n$ the total number of brain regions. As for the estimation of DFC, instantaneous phases were calculated from analytic signals. For empirical data, MS was estimated for each subject separately and subsequently averaged. In the model it was obtained from the full-length signal. The difference between average empirical MS and model MS reflects how well the model reproduces this metric.

\section{Optimization of the local control variable}

The region specific bifurcation parameter $a_{j}$ was optimized by fitting spectral information of the empirical BOLD signals in each region (see Fig. $1 \mathrm{~b}$ for a schematic overview). Specifically, local parameters were tuned to reproduce the region-specific proportions of power in a narrow band $(0.04-0.07 \mathrm{~Hz})$ with respect to a broad band $(0.04-0.25 \mathrm{~Hz})$. To do so, the power spectrum $P_{j}(f)$ for each node $j$ in the narrow as well as in the broad band was calculated to obtain the proportion

$p_{j}=\frac{\int_{.04}^{.07} P_{j}(f) d f}{\int_{.04}^{.25} P_{j}(f) d f}$

Subsequently, the local bifurcation parameter was updated according to a gradient descendent strategy

$a_{j}=a_{j}+\eta\left(p_{j, \text { empirical }}-p_{j, \text { simulated }}\right)$

A learning rate of $\eta=.1$ was used. Adjustment of this local parameter was only carried out after global parameters had been fit to assure that the procedure started with values in the vicinity of a global optimum. As for the optimization of global control variables, adjustment of the local bifurcation parameter was based on group data.

\section{Results}

\section{Rich club}

Rich club regions were identified from a binarized SC matrix obtained from setting all its non-zero entries to one. From this binary adjacency matrix rich club coefficients were calculated as the fraction of the number of existing connections between regions with degree larger than $k$ to the possible number of connections among these regions (Colizza et al., 2006; Zhou and Mondragon, 2004). Next, the statistical significance of rich club coefficients for each degree $k$ was determined by calculating the rich club coefficients for a set of 1000 degreepreserving rewired adjacency matrices (Maslov and Sneppen, 2002) and identifying the first $k$ for which the rich club coefficient of the binarized SC was larger than the 95th percentile of the rich club coefficients corresponding to the rewired matrices (see Fig. 3). Candidate rich club regions were subsequently identified as those whose degree exceeded the first $k$ level for which the rich club coefficient reached statistical significance. These candidate regions included the bilateral precuneus, the bilateral superior frontal cortex, the bilateral superior parietal cortex, and the right insula. To ensure that these regions were not only individually rich but also formed a dense club we calculated the internal density of this set of regions. Subsequently, we, in turn, removed each region and re-evaluated the internal density of the remaining set. If a region is part of the rich club its removal should hardly affect internal density. On the other hand, if a region is not part of the rich club its removal should lead to a sharp rise in density. Removal of the left precuneus, the right superior frontal cortex, left superior parietal, and right superior parietal each lead to decreases in internal density by $6.67 \%$. Removal of the right precuneus and left superior frontal cortex each lead to a slight increase in internal density by $2.67 \%$. These regions, therefore, form a dense club. 


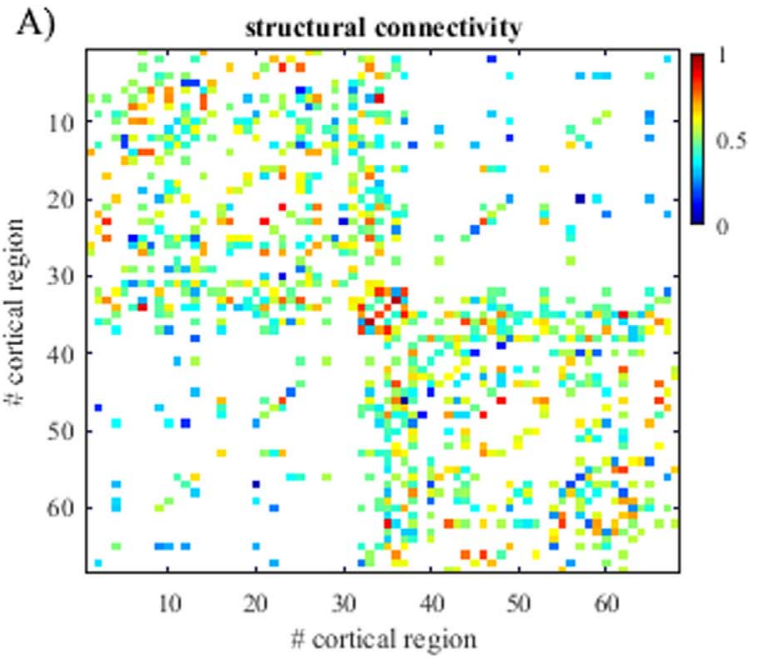

B)

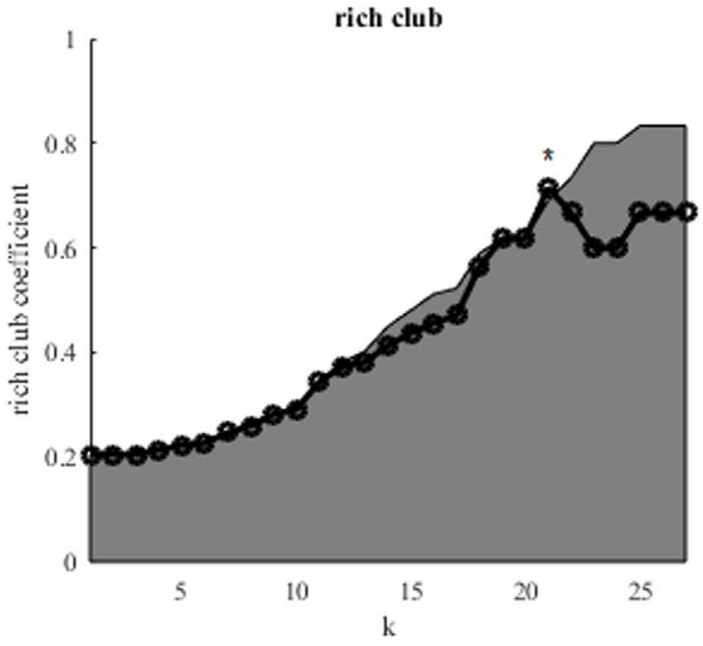

Fig. 3. Structural data. Panel A) shows the weighted structural connectivity matrix consisting of 68 cortical regions. Regions are arranged according to the ordering given in Table 1 with the rich club in the center of the matrix. Panel B) shows the rich club coefficient of the structural connectivity matrix as a function of degree cutoff $k$ (black line). The shaded region depicts the threshold the rich club coefficient needs to exceed to reach significance. Significance was reached for $k=21$ (marked with an asterisk).

However, removal of the right insula lead to an increase in internal density by $21.33 \%$. Since ninety-five percent of density changes lay within $\pm 8.31 \%$ and density changes produced by removal of the right insula fell outside this interval, it was not considered a rich club region in this study. We validated this decision by removing the right insula from the SC matrix and observing no changes with regard to the rich club phenomenon. While exclusion of the right insula from the rich club was thus justified in our study, other studies using a different data set or employing a different parcellation scheme might still identify it as part of the rich club.

\section{Whole-brain functional dynamics and model fitting}

Fig. 4 shows the group level FC (A), DFC (B), and MS (C) observed for rest as well as for the four tasks which the model needed to reproduce. As can be appreciated from the figure, whole-brain FC patterns were highly similar. Indeed, all pairs correlated strongly with Pearson correlations in the range from .84 (rest with mental rotation) to .88 (flanker with mental rotation). Dynamic FC representing the dissolution and re-assembly of short-lived functional connectivity patterns showed differences between resting and task states as well as among different task states. The distributions of cosine similarities (see methods for details) were significantly different according to a paired Kolmogorov-Smirnoff test for all pairs of rest to task as well as among tasks (P-values for all paired tests were «.001). Finally, metastability did not differ between resting and task states nor among different task states with the lowest P-value in a paired t-test equal to .26 (rest vs n-back).

In order to find the optimal parameter settings for each task, we performed an exhaustive search of the parameter space. We examined global coupling $G$ in the range from zero to .175 in 100 steps and global bifurcation parameter $A$ in the range from minus to plus .5 in 30 steps. For all functional states, Fig. 5 shows the Pearson correlation between model and empirical FC (A), the Kolmogorov-Smirnoff distance between model and empirical DFC (B), and model MS (C) for the entire parameter space (i.e. all combinations of explored values for $A$ and $G$ ). Supplementary Figs. 3-5 give a more detailed account of the three metrics obtained from simulations at different working points for resting state data. To obtain an overall measure of how well our simulations reproduce empirical data, we combined the three metrics. First we converted each metric to a distance between model and empirical data. For FC this involved subtracting the Pearson correlation from one. For MS this involved computing the difference between model and empirical MS values. The Kolmogorov-Smirnoff distance remained unchanged. Next we normalized each metric with respect to its range to adjust for differences in scales. Finally, we averaged across these three normalized distance measures (Fig. 5d). Alternatively, the maximum across the normalized distance measures might be taken. Optimal parameter values correspond to the region where this global measure is minimized. We identified the same region using the average and the maximum to aggregate distance measures. This region was highly similar across tasks with a broad range of global coupling values but only a narrow band of bifurcation parameter values leading to good model fit. The latter was especially due to the Kolmogorov-Smirnoff distance between model and empirical DFC distributions as well as MS. In both resting and all task states, we found the optimal value for $A$ to be just below zero implying that overall brain regions operated close to a transition from asynchronous to oscillatory dynamics (i.e., at the brink of a supercritical Hopf bifurcation) for all functional states.

\section{Local dynamics}

Next, we continued with the estimation of local bifurcation parameters $a_{j}$ for all regions $j$ within each functional state. Given the broad range of good global coupling values, we chose to estimate local bifurcation parameters for each task for a number of optimal global coupling values ranging from .04 to .16. However, since results were not qualitatively affected by this choice and since global coupling is conceptually a non-changing structural scaling factor, we present results only for the higher end of the range $(G=.16)$. Local bifurcation parameter values were initialized to the average of optimal global bifurcation parameters values obtained across the range of optimal global coupling ( $a_{j}=A_{\text {opt }}=-.0517$ ) thus ensuring that values were in the vicinity of a global optimum before starting the gradient descent. To evaluate the robustness of our local optimization procedure we also initialized $a_{j}$ to random values in the range from - .5 to .5 (the results can be found in supplementary Fig 6).

The results of local parameter estimations are shown in Fig. 6. For resting state, $60 \%$ of peripheral regions present with a bifurcation parameter above zero. As can be seen from the figure, rich club regions present with negative bifurcation parameters during rest. During task performance the number of peripheral regions presenting with a positive bifurcation parameter decreases. For the n-back, flanker, mental rotation, and odd-man out tasks $30 \%$, 44\%, 34\%, and $44 \%$ of peripheral regions present with a positive bifurcation parameter, respectively. The mean bifurcation parameter across peripheral regions was $\bar{a}_{p}=-.03$ (95\% CI $\left.[-.10, .04]\right)$ for rest, $\bar{a}_{p}=-.31$ (95\% CI $[-.41$, $-.20])$ for the n-back task, $\bar{a}_{p}=-.13$ (95\% CI [-.19, -.07]) for the flanker 
A) $\mathrm{FC}$
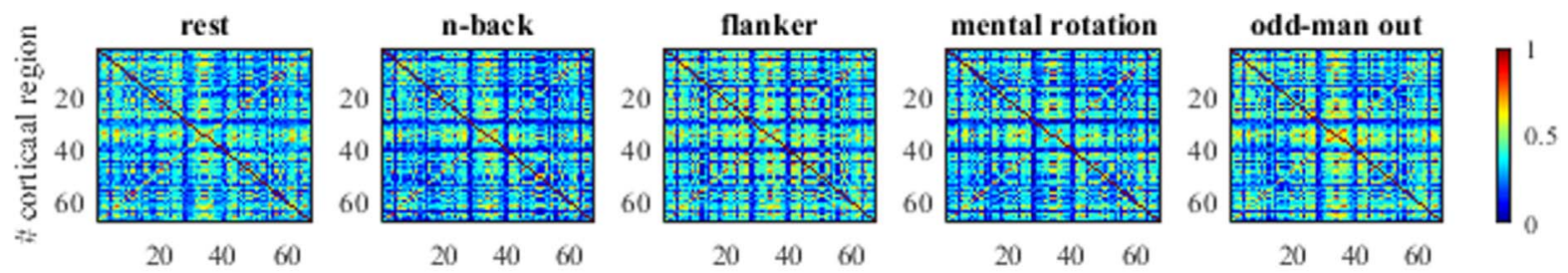

B)

\# cortical region
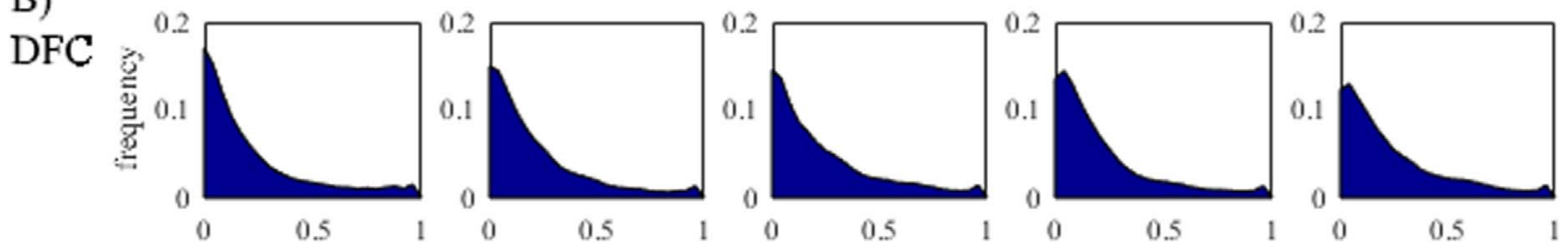

C)

cosine similarity

MS
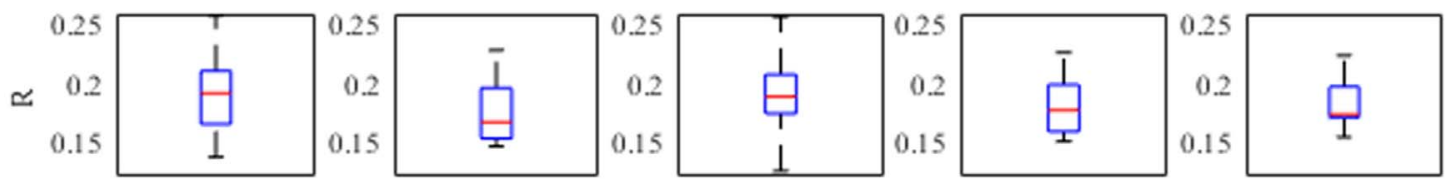

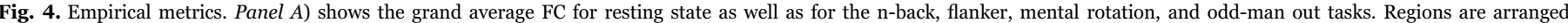

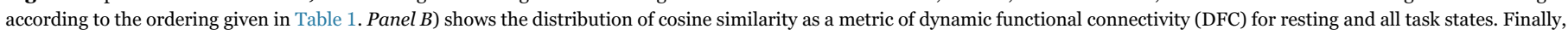
panel $C$ ) shows boxplots of the standard deviation of the Kuramoto order parameter $R$ as a metric of metastability observed in all functional states.
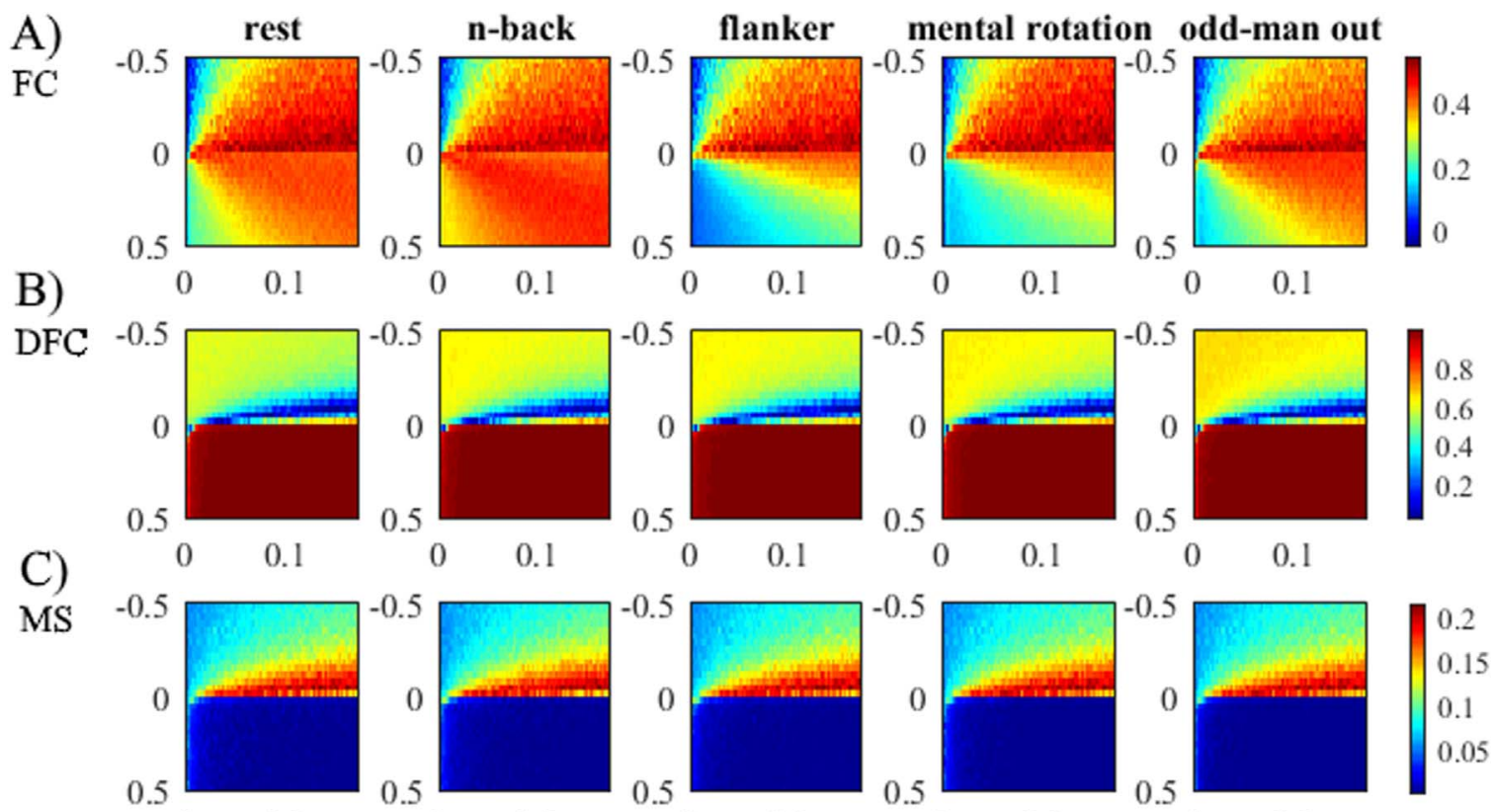

D)
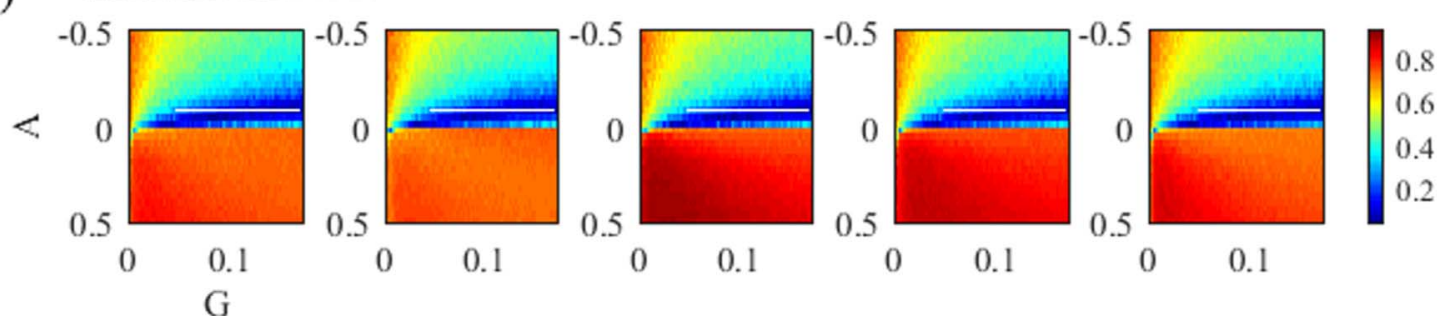

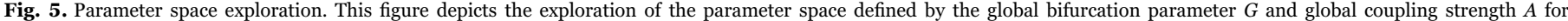

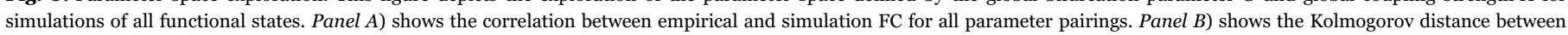

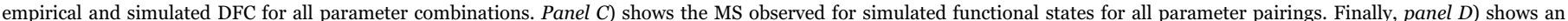

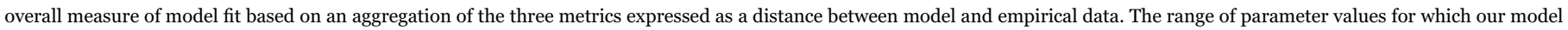
simulations where performed is marked in white. We report results only for $G=.16$ and $A=-.0517$. 

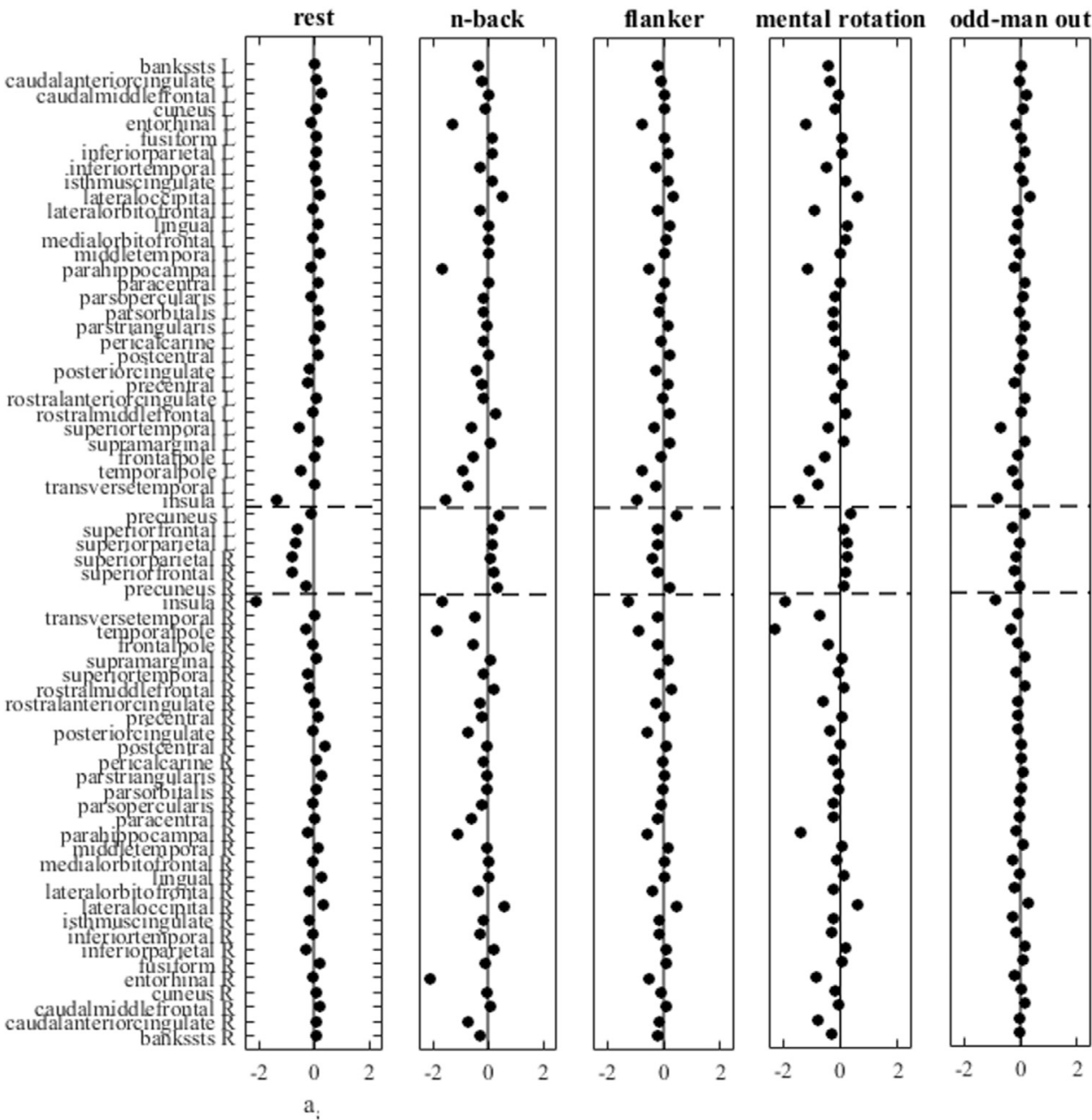

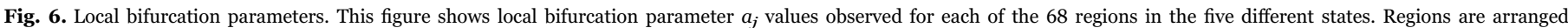

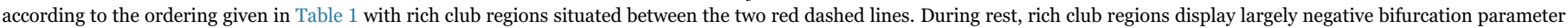

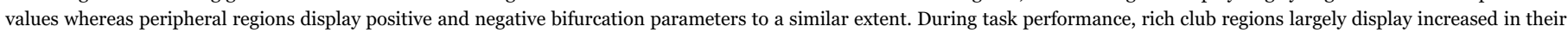
local bifurcation parameter values as compared to rest. (For interpretation of the references to color in this figure legend, the reader is referred to the web version of this article.)

task, $\bar{a}_{p}=-.30$ (95\% CI $\left.[-.41,-.20]\right)$ for the mental rotation task, and $\bar{a}_{p}=-.05$ (95\% CI $\left.[-.10,-.01]\right)$ for the odd-man out task. While overall bifurcation parameter values drop for task states as compared to rest, rich club regions present with higher bifurcation parameters during task performance. The mean bifurcation parameter across rich club regions was $\bar{a}_{r c}=-.53$ (95\% CI $\left.[-.59,-.48]\right), \bar{a}_{r c}=.22$ (95\% CI $[.20$, $.25]), \bar{a}_{r c}=-.05$ (95\% CI $\left.[-.12, .01]\right), \bar{a}_{r c}=.21$ (95\% CI $\left.[.19, .23]\right)$, and $\bar{a}_{r c}=-.09(95 \% \mathrm{CI}[-.12,-.06])$ for rest and the four tasks, respectively. To further test whether rich club regions exhibit stronger positive changes in their bifurcation parameter than other cortical regions, we performed a cluster-based bootstrapping procedure. To that end we obtained the differences in bifurcation parameter values between task and rest for each region and computed the average difference across the set of rich club regions. Subsequently, we repeatedly (1000 times) sampled six regions from the entire set of cortical regions with replacement and calculated the average change within those sample. For all tasks the set of rich club regions exhibits changes far exceeding the 99th percentile of the thusly generated null-distribution. Specifically, the set of rich club regions presented with an average change of $.75, .48, .74$, and .45 for the n-back, flanker, mental rotation, and odd-man out tasks with their respective 99th percentiles being equal to $.27, .28, .28$, and .30 . Supplementary Fig. 7 show a ranking of brain regions according to the changes they exhibit in local bifurcation parameters per task. Next, we evaluated in how far individual rich club regions presented with an increased bifurcation parameter during task performance as compared to rest. We evaluated changes at the individual regions level by performing a blocked bootstrap test. The null-distribution for each region in each task was created from 1000 simulations of randomly generated rest and task samples and calculating the difference (task - rest) between local bifurcation parameters. Samples were created by first randomly drawing from the subject pool with replacement and then randomly placing one of each subject's two states in the rest and the other in the task sample repeatedly until each sample comprised 14 subjects. Local bifurcation parameters were then optimized in each of these samples. Fig. 7 shows the results. For the nback task all rich club regions exhibited a difference (rest-task) exceeding the 95th percentile of the bootstrap null-distribution. Furthermore, the difference observed for the left and right superior parietal cortices and the right superior frontal cortex exceeded the $99^{\text {th }}$ percentile. For the flanker task differences observed in the left and right precuneus as well as the left and right superior parietal cortices exceeded the 95th percentile. Differences observed in the left and right precuneus exceeded the $99^{\text {th }}$ percentile. For the mental rotation task differences in all but the left superior frontal cortex exceeded the $95^{\text {th }}$ percentile and the right superior frontal cortex exceeded the 99th percentile. Finally, for the odd-man out task only differences observed for the left and right superior parietal cortices exceeded the 95th percentile while none exceeded the 99th percentile. The cluster analysis clearly indicates that rich club regions as a group exhibited more oscillatory behavior during task performance as compared to rest. 

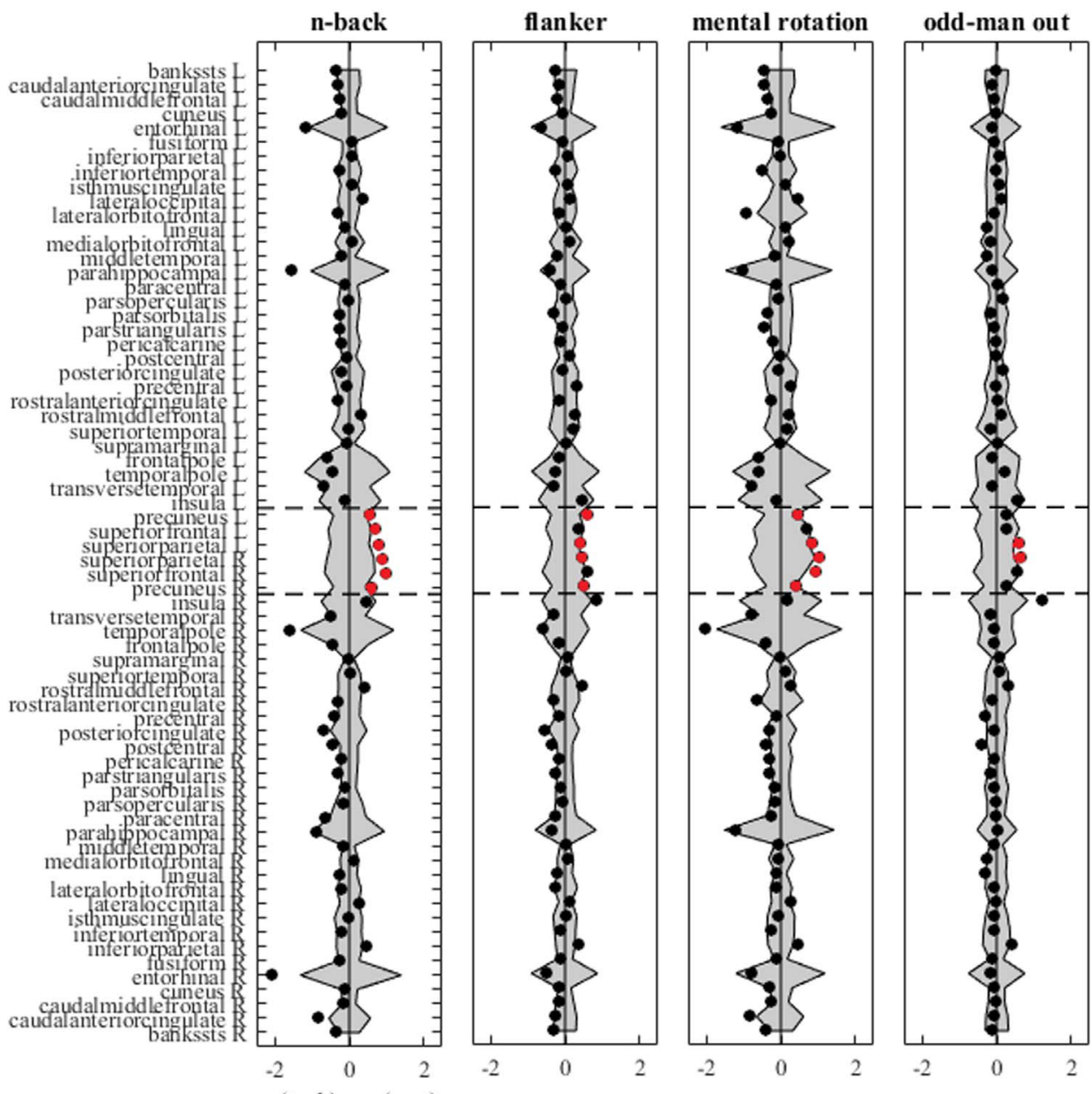

$\mathrm{a}_{\mathrm{i}}($ task $)-\mathrm{a}_{\mathrm{i}}$ (rest)

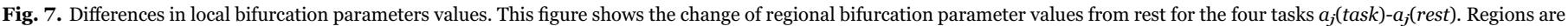

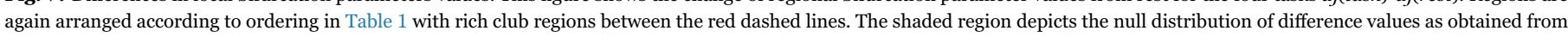

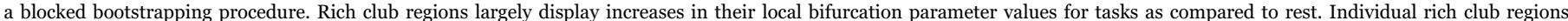

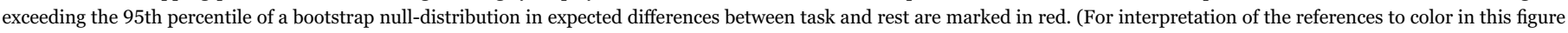
legend, the reader is referred to the web version of this article.)

However, the analyses of individual rich club regions shows that which rich club regions increased their oscillatory behavior and to what degree was task dependent.

Following up on these results we investigated whether cortex-wide functional connectivity was indeed related to oscillations. First, we examined the general effect of oscillations on simulated functional connectivity by calculating average global connectivity resulting from simulations in which all regions exhibited identical bifurcation parameters ranging from -.25 to +.05 with global coupling fixed at $G=.16$. Increasing the bifurcation parameter was accompanied by increased correlations between pairs of brain regions (see Fig. 8). Furthermore, positive bifurcation parameter values were associated with very strong correlations. Note that bifurcation parameter values just below zero; that is, at the optimal point determined previously, showed the largest range of correlations reflecting the vast dynamic range the system exhibited at this point. Subsequently, we identified for each functional state the subset of regions whose bifurcation parameter was estimated to be above zero as well as the subset of regions whose bifurcation parameter was estimated to be below zero, and calculated the average empirical functional connectivity within these two subsets. We obtained bifurcation parameters and functional connectivity separately by repeatedly (100 times) splitting the data in half and using one half of the data for the estimation of each. Fig. 8b shows boxplots of the resulting estimates of average FC for the subsets of oscillating and non- oscillating regions for each functional state. In agreement with the finding that oscillations increased simulated functional connectivity values, average empirical $\mathrm{FC}$ was indeed higher among the subset of regions presenting with a positive bifurcation parameter for all functional states.

Finally, we used the model to investigate whether oscillations exhibited by cortical rich club regions can be more effective in bringing about functional coupling than oscillations exhibited by peripheral regions alone. To this end we investigated in how far a set of brain regions exhibiting oscillatory behavior can synchronize cortical activation across the entire network. Two factors were of interest in this analysis: the first was the size of the set of brain regions exhibiting oscillatory behavior, whereas the second was whether this set included the rich club. The first factor was included since a sufficiently large number of peripheral (i.e. non rich club) regions exhibiting oscillations might be able to bring about synchronization without assistance of an oscillating rich club (as should be the case during rest). In terms of the first factor (size), a set comprising of $12,18,24$, or 30 randomly selected peripheral regions were assigned a positive bifurcation parameter value $\left(a_{p o s}=.5\right)$ while the remaining regions were assigned a negative bifurcation parameter value $\left(a_{\text {neg }}=-.5\right)$. This constituted at the same time the condition of only peripheral regions exhibiting oscillations. For the condition in which the rich club was among the set of oscillating regions, six randomly selected peripheral regions among 

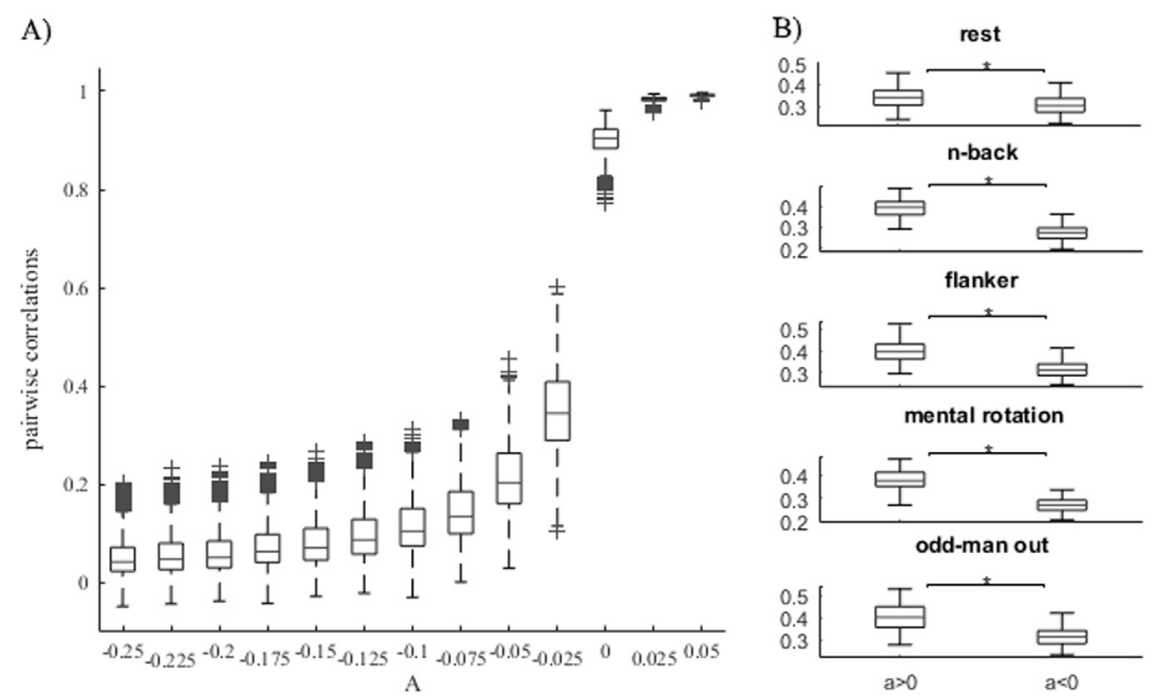

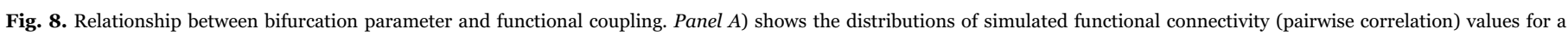

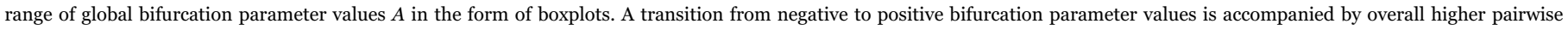

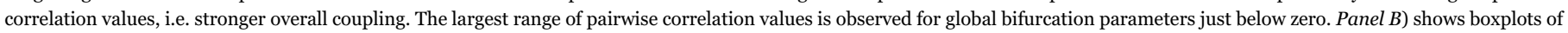

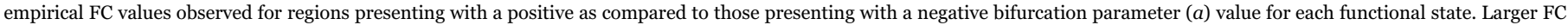
values are observed for brain regions presenting with positive as compared to negative bifurcation parameter values, irrespective of the functional sate.

the set of oscillating regions were replaced by rich club regions; i.e. the bifurcation parameter of these selected regions was set to - .5 while the bifurcation parameter of all rich club regions was set to +.5. All peripheral regions had characteristic frequencies in the range from $.04 \mathrm{~Hz}$ to $.07 \mathrm{~Hz}$ whereas rich club regions were assigned a characteristic frequency at the center of this range $(.055 \mathrm{~Hz})$ in order to provide a pulse frequency. Each region's phase was initialized to zero but was effectively determined by the additive Gaussian noise term in Eq. (1). We measured synchrony among brain regions both in terms of phase and in terms of frequency. For synchrony in terms of phase we calculated the Kuramoto order parameter $R$ (see methods for details) across brain regions at a frequency of $.055 \mathrm{~Hz}$. In terms of frequency we measured synchrony by calculating the power spectrum for each region in the aforementioned range normalized with respect to its maximum value. Subsequently, we averaged the power spectra over regions. If all brain regions exhibit fluctuations at the same frequency, the average power spectrum is characterized by a single sharp peak at the shared frequency $(\sim .055 \mathrm{~Hz})$. If, on the other hand, brain regions exhibit fluctuations at different frequencies, the average power spectrum is characterized by a broader peak. In order to characterize the breadth of the peak we fitted a Gaussian distribution to it and calculated its FWHM relative to the considered frequency range. We performed these simulations for 100 random selections of peripheral regions to exhibit oscillations nested in 100 randomly generated distributions of characteristic frequencies across regions. Hence, we performed 10,000 simulations for each combination of the number of oscillating peripheral regions and an oscillating or not oscillating rich club.

Fig. 9 shows the relative FWHM as well as the Kuramoto order parameter $R$ as a function of the total number of oscillating brain regions separately for the case that rich club regions exhibited oscillations and for the case that they did not. Synchronization both in terms of frequency as well as in terms of phase increased with a larger number of oscillating regions. Importantly, an oscillating rich club accelerated this effect. This effect was most pronounced for phase synchronization, but also apparent for frequency synchronization; especially if few peripheral regions were supplemented by the rich club. Such a configuration was characteristic of task performance. A larger number of oscillating peripheral regions (30 in this simulation) achieved good synchronization levels even in the absence of an oscillating rich club.

\section{Discussion}

Using a combined computational modeling and fMRI approach we investigated whether oscillations constitute a viable candidate mechanism through which cortical rich club regions can support functional network formation. Our results support this idea. First, brain regions whose local bifurcation parameter suggested that they exhibit oscillations showed an increased propensity to engage in functional coupling; both in terms of empirical as well as simulated functional connectivity. Second, in simulations, oscillations exhibited by the rich club provided a timing signal sufficient to synchronize brain regions operating at a range of idiosyncratic frequencies and phases. Finally, increased oscillatory behavior exhibited by rich club regions with simultaneous reductions in the number of peripheral regions engaging in oscillations demarcated empirically observed task states from resting state. In the present study we used tasks reflecting a wide range of cognitive functions; namely, working memory, executive function and inhibition, mental rotation, and semantic reasoning. Given that these cognitive functions are not only conceptually distinct but minimally overlapping with respect to their associated functional networks (Smith et al., 2009), it is reasonable to conclude that the observed changes in oscillatory behavior among the rich club as a whole are general rather than limited to specific cognitive processes. At the same time, differences in the degree to which individual rich club regions exhibit oscillations indicates that this behavior is adaptive to task demands. During task performance stable coupling among functionally relevant, specialized brain regions is required. During task performance, oscillating rich club regions might thus provide a pulse frequency to facilitate functional coupling among functionally relevant brain regions involved in task execution. Specifically, the reduced number of oscillating peripheral regions (compared to rest) might be insufficient to sustain stable functional coupling unless supported by oscillations exhibited by rich club regions. This would be in line with previous simulation findings that the rich club can support functional coupling among peripheral brain regions (Gollo et al., 2015; Schmidt et al., 2015; Senden et al., 2014). During rest, on the other hand, rich club regions were not oscillating and could thus not impose a specific rhythm on the cortex. This behavior might be more conducive to flexible re-coupling among peripheral regions since it allows for larger groups of peripheral regions to engage in oscillatory behavior and hence to engage in (potentially short-lived) functional coupling without 

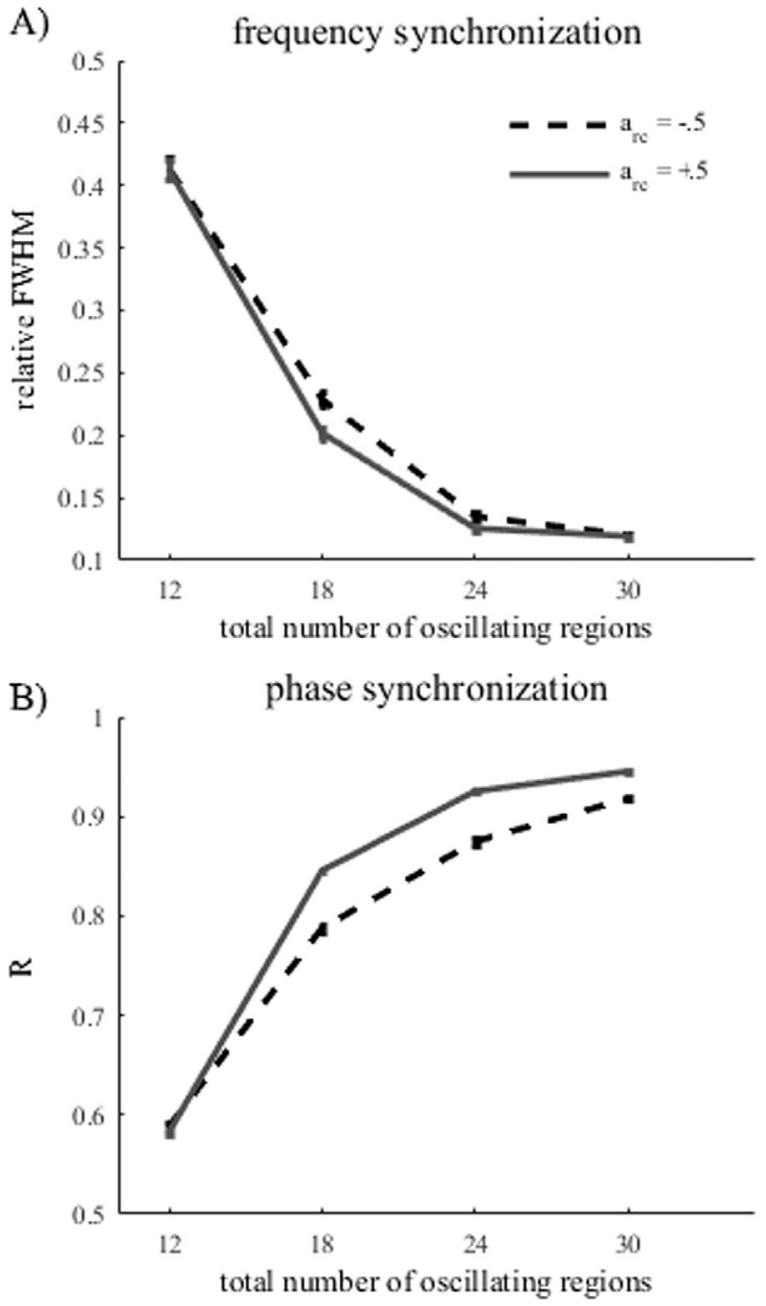

Fig. 9. Synchronization. This figure depicts the extent to which brain regions are synchronized as a function of the number of brain regions set to have a positive bifurcation parameter value. Separate lines represent the cases where the set of regions having a positive bifurcation parameter value include the rich club (green) or do not include the rich club (red). Panel $A$ ) shows the extent of synchronization in terms of frequency as measured by the full width at half maximum of the power spectrum averaged across brain regions. Lower values indicate better synchronization. For the range of $\sim 14-24$ regions presenting with a positive bifurcation parameter value, it is beneficial if this set includes the rich club. Panel B) shows the extent of synchronization in terms of phases as measured by the Kuramoto order parameter $R$. Larger values indicate better synchronization. Unless a large number of regions is oscillating it is beneficial for synchronization to include the rich club among the oscillating regions. (For interpretation of the references to color in this figure legend, the reader is referred to the web version of this article).

producing a fully coupled (epileptic) state. This might be advantageous for the exploration of the brain's functional repertoire (Deco and Jirsa, 2012).

A question at this point is whether the rich club is the origin of this coordination or provides the means for peripheral regions to exhibit control over the network. Previous research suggests the latter since the states of a complex system, and especially those which are difficult to reach, are controlled by regions of low degree rather than by hubs (Gu et al., 2015; Liu et al., 2011). Nevertheless, the presence of hubs largely increases the general controllability of said system (Liu et al., 2011). Note that controllability of networks in general does not necessarily require rich club organization but rather some densely interconnected structural backbone (e.g. a single hyperconnected node). In the brain this backbone is provided by the cortical rich club (van den Heuvel et al., 2012) whose role might be to enable peripheral regions to control the state, i.e. functional network configuration, of the cortex. As such, the rich club might provide a central workspace of information integration wherein peripheral brain regions compete for control of the system as recently proposed by Shanahan (2012). During rest, such conflict might be unresolved or, at least, recurring whereas task performance might necessitate a clear winner. This winner might then recruit the rich club to move the brain into the required functional state as well as to support communication among regions constituting this state. Our results suggest that this is dynamically achieved by moving the rich club into an (infraslow) oscillatory regime.

Our as well as previous findings (Gollo et al., 2015; Hiltunen et al., 2014; Monto et al., 2008; Schmidt et al., 2015; Vanhatalo et al., 2004) suggest that oscillations are relevant for global brain communication. However, it is currently unknown whether infraslow oscillations are immediately relevant for functional integration or are merely the filtered manifestation of a faster frequency band. It is principally conceivable, for instance, that stimuli presented at regular intervals impose an oscillatory activation pattern on task-relevant brain regions. While inter-trial intervals do not vary within tasks in our study, they differ between tasks thus reducing the probability that our results are due to the stimulation protocol. Furthermore, neither stimulation frequency aliases into the frequency band of interest $(.04 \mathrm{~Hz}-0.7 \mathrm{~Hz})$. Finally, if the emergence of oscillations in our simulations were due purely to stimulation, we would expect largest changes in oscillatory behavior among task-specific and sensory regions rather than among rich club regions. These considerations do not rule out, however, that the observed infraslow oscillations constitute a proxy for oscillations occurring within alpha/beta or gamma frequency bands. This is a question that needs to be addressed using empirical data of higher temporal resolution. Due to the slow time scale of infraslow oscillations, it is at any rate unlikely that they carry the actual signals to be communicated between brain regions. A more likely function for them would be to align excitability states of brain regions (Hiltunen et al., 2014; Vanhatalo et al., 2004) and hence to establish communication channels among them. Such an interpretation for the potential role of infraslow oscillations would agree with recent electrophysiological findings which showed that the power envelope of higher frequencies, most prominently in the range from $8 \mathrm{~Hz}$ to $32 \mathrm{~Hz}$, is itself modulated at infraslow frequencies (Hipp et al., 2012). These higher frequencies are far more likely to reflect the actual signal in line with a communication through coherence hypothesis (Fries, 2001, 2009; Michalareas et al., 2016). While our findings thus speak to the potential function of rich club regions to provide a timing signal for global brain communication, it remains to be investigated whether rich club regions also mediate and/or modulate high frequency signals exchanged among peripheral regions.

Furthermore, if infraslow oscillations are indeed meaningful, a question arises regarding the neural mechanism giving rise to them. The neural mass model employed here gives a descriptive rather than a mechanistic account of local dynamics. This provides the appropriate level of detail for investigating the question of which local dynamics are exhibited by interconnected brain regions and how these change from resting to task states in an fMRI context. However, future work is needed to address the neural mechanism underlying these local dynamics. A candidate mechanism comes in the form of interactions between cortical and thalamic neuronal populations (Breakspear et al., 2006; Freyer et al., 2011, 2012). A neural mass model implementing these interactions in the presence of state-dependent noise has recently been shown to account for known spontaneous transitions between two distinct modes of power in the alpha frequency band (Freyer et al., 2009, 2011). Given the relationship between infraslow oscillations and the alpha and beta rhythms (Hipp et al., 2012), the slow dynamics examined here might reflect changes in the power envelope resulting from these transitions. Finally, since relay nuclei in the thalamus can themselves exhibit infraslow oscillations (Lórincz et al., 2009), a straightforward extension of the cortico-thalamic neural mass model may allow it to account for both spontaneous as well as structured, i.e. 
infraslow oscillatory, transitions between low and high power modes in the alpha and/or beta bands.

Apart from the above, our results lead to a number of further questions and predictions. First, if the rich club is indeed a locus of competition among peripheral regions for network control, then it should receive a high degree of input but only provide sparse output; namely output reflecting communication among the winning set of regions. Furthermore, this should be task dependent both in terms of the total amount of input and output each rich club region receives and sends as well as in terms of the exact patterns of in and out relations. Second, if coupling via oscillations reflects the establishment of taskrelevant communication channels, then one should expect to find information transfer among these regions (possibly mediated through the rich club) coded at higher frequencies, and that the informational content of these signals is relevant for, and reflects, the task at hand. Related to this, the information content as well as the degree to which rich club regions exhibit oscillatory behavior might also reflect task difficulty, a factor we did not take into account here. Finally, an important question future research should address is how local and whole-brain dynamics vary across individuals and how this relates to individual differences in the underlying structural connectivity. This would call for fMRI and diffusion-weighted MRI data to be acquired in the same subjects. Additionally, a larger sample size would be helpful in characterizing this variability. Furthermore, fitting model parameters to individual subject data would require longer runs thus putting practical limits on the number of tasks for which data can be acquired.

In conclusion, we find that (infraslow) oscillations constitute a potential mechanism for rich club regions to shape whole-brain functional coupling in a functionally specific manner. In conjunction with converging results from connectomics (van den Heuvel et al., 2012; Zamora-López et al., 2009), neuroimaging (Braga et al., 2013; Leech et al., 2012), and computational modeling (Deco and Jirsa, 2012; Deco et al., 2012; Gollo et al., 2015; Gu et al., 2015; Liu et al., 2011; Schmidt et al., 2015; Senden et al., 2014), this is in line with the notion that rich club regions support and shape the concerted interplay of specialized brain regions required for higher cognition. This leads to a conceptualization of the rich club as the structural underpinning of a global neuronal workspace (Dehaene and Changeux, 2011; Harriger et al., 2012; Shanahan, 2012). Furthermore, our results emphasize the importance of the integration of large-scale computational models with locally specific dynamics in conjunction with empirical research for investigating whole-brain functional network states. While all empirical metrics could be reproduced by our model using identical global parameters for each task, fitting and interpretation of parameters controlling local dynamics enabled us to demarcate resting and task states.

\section{Acknowledgements}

Authors MS and RG were supported by the European Research Council under the European Union's Seventh Framework Programme (ERC-2010-AdG, ERC grant agreement no. 269853). Author GD was supported by the ERC Advanced Grant: DYSTRUCTURE (no. 295129), by the Spanish Research ProjectSAF2010-16085 and by European Community's Seventh Framework Programme under the project "BrainScales" (project number 269921). Author MPvdH was supported by a VENI grant of The Netherlands Organization for Scientific Research (NWO) (451-12-001) and by a Fellowship of the Brain Center Rudolf Magnus. The authors declare no competing financial interests.

\section{Appendix A. Supplementary material}

Supplementary data associated with this article can be found in the online version at http://dx.doi.org/10.1016/j.neuroimage.2016.10. 044.

\section{References}

Baars, B.J., 2005. Global workspace theory of consciousness: toward a cognitive neuroscience of human experience. Boundaries Conscious.: Neurobiol. Neuropathol. 150, 45-53.

Bear, M.F., Connors, B., Paradiso, M., 2006. Neuroscience: Exploring the Brain 3rd Revise. Lippincott Williams and Wilkins, Baltimore.

Braga, R.M., Sharp, D.J., Leeson, C., Wise, R.J., Leech, R., 2013. Echoes of the brain within default mode, association, and heteromodal cortices. J. Neurosci. 33 (35), $14031-14039$.

Breakspear, M., Roberts, J.A., Terry, J.R., Rodrigues, S., Mahant, N., Robinson, P.A., 2006. A unifying explanation of primary generalized seizures through nonlinear brain modeling and bifurcation analysis. Cereb. Cortex 16 (9), 1296-1313. http:// dx.doi.org/10.1093/cercor/bhj072.

Cabral, J., Hugues, E., Deco, G., 2011. Simulated functional networks in health and schizophrenia: a graph theoretical approach. BMC Neurosci. 12 (Suppl 1), P63.

Cabral, J., Luckhoo, H., Woolrich, M., Joensson, M., Mohseni, H., Baker, A., Deco, G., 2014. Exploring mechanisms of spontaneous functional connectivity in MEG: how delayed network interactions lead to structured amplitude envelopes of band-pass filtered oscillations. Neuroimage 90, 423-435.

Cole, M.W., Reynolds, J.R., Power, J.D., Repovs, G., Anticevic, A., Braver, T.S., 2013 Multi-task connectivity reveals flexible hubs for adaptive task control. Nat. Neurosci. 16 (9), 1348-1355.

Colizza, V., Flammini, A., Serrano, M.A., Vespignani, A., 2006. Detecting rich-club ordering in complex networks. Nat. Phys. 2 (2), 110-115.

Damasio, A.R., 1989. Time-locked multiregional retroactivation: a systems-level proposal for the neural substrates of recall and recognition. Cognition 33 (1), 25-62.

de Reus, M.A., van den Heuvel, M.P., 2013. Estimating false positives and negatives in brain networks. NeuroImage 70, 402-409. http://dx.doi.org/10.1016/ j.neuroimage.2012.12.066.

de Reus, M.A., van den Heuvel, M.P., 2014. Simulated rich club lesioning in brain networks: a scaffold for communication and integration? Front. Human. Neurosci. 8. http://dx.doi.org/10.3389/fnhum.2014.00647.

Deco, G., Jirsa, V.K., 2012. Ongoing cortical activity at rest: criticality, multistability, and ghost attractors. J. Neurosci. 32 (10), 3366-3375. http://dx.doi.org/10.1523/ JNEUROSCI.2523-11.2012.

Deco, G., Jirsa, V.K., McIntosh, A.R., 2011. Emerging concepts for the dynamical organization of resting-state activity in the brain. Nat. Rev. Neurosci. 12 (1), 43-56. http://dx.doi.org/10.1038/nrn2961.

Deco, G., Senden, M., Jirsa, V., 2012. How anatomy shapes dynamics: a semi-analytical study of the brain at rest by a simple spin model. Front. Comput. Neurosci.. http:// dx.doi.org/10.3389/fncom.2012.00068.

Dehaene, S., Naccache, L., 2001. Towards a cognitive neuroscience of consciousness: basic evidence and a workspace framework. Cognition 79 (1), 1-37.

Dehaene, S., Changeux, J.-P., 2011. Experimental and theoretical approaches to conscious processing. Neuron 70 (2), 200-227.

Desikan, R.S., Ségonne, F., Fischl, B., Quinn, B.T., Dickerson, B.C., Blacker, D., et al., 2006. An automated labeling system for subdividing the human cerebral cortex on MRI scans into gyral based regions of interest. Neuroimage 31 (3), 968-980.

Eriksen, B.A., Eriksen, C.W., 1974. Effects of noise letters upon the identification of a target letter in a nonsearch task. Percept. Psychophys. 16 (1), 143-149.

Flourens, P., 1842. Recherches expérimentales sur les propriétés et les fonctions du système nerveux dans les animaux vertébrés. Ballière.

Flowers, K.A., Robertson, C., 1985. The effect of Parkinson's disease on the ability to maintain a mental set. J. Neurol. Neurosurg. Psychiatry 48 (6), 517-529.

Freyer, F., Aquino, K., Robinson, P.A., Ritter, P., Breakspear, M., 2009. Bistability and non-gaussian fluctuations in spontaneous cortical activity. J. Neurosci. 29 (26), 8512-8524. http://dx.doi.org/10.1523/JNEUROSCI.0754-09.2009.

Freyer, F., Roberts, J.A., Becker, R., Robinson, P.A., Ritter, P., Breakspear, M., 2011. Biophysical mechanisms of multistability in resting-state cortical rhythms. J. Neurosci. 31 (17), 6353-6361.

Freyer, F., Roberts, J.A., Ritter, P., Breakspear, M., 2012. A canonical model of multistability and scale-invariance in biological systems. PLoS Comput. Biol. 8 (8), e1002634.

Fries, P., 2001. Rapid feature selective neuronal synchronization through correlated latency shifting. Nat. Neurosci. 4 (2), 194.

Fries, P., 2009. Neuronal gamma-band synchronization as a fundamental process in cortical computation. Annu. Rev. Neurosci. 32 (1), 209-224. http://dx.doi.org/ 10.1146/annurev.neuro.051508.135603.

Ghosh, A., Rho, Y., McIntosh, A.R., Kötter, R., Jirsa, V.K., 2008. Noise during rest enables the exploration of the brain's dynamic repertoire. PLoS Comput. Biol. 4 (10), e1000196.

Glasser, M.F., Sotiropoulos, S.N., Wilson, J.A., Coalson, T.S., Fischl, B., Andersson, J.L. et al., 2013. The minimal preprocessing pipelines for the Human Connectome Project. Neuroimage 80, 105-124.

Glerean, E., Salmi, J., Lahnakoski, J.M., Jääskeläinen, I.P., Sams, M., 2012. Functional magnetic resonance imaging phase synchronization as a measure of dynamic functional connectivity. Brain Connect. 2 (2), 91-101.

Gollo, L.L., Zalesky, A., Hutchison, R.M., van den Heuvel, M., Breakspear, M., 2015. Dwelling quietly in the rich club: brain network determinants of slow cortical fluctuations. Philos. Trans. R. Soc. Lond. B: Biol. Sci. 370 (1668), 20140165.

Grigg, O., Grady, C.L., 2010. Task-related effects on the temporal and spatial dynamics of resting-state functional connectivity in the default network. PLoS One 5 (10), [e13311-e13311].

Gu, S., Pasqualetti, F., Cieslak, M., Telesford, Q.K., Alfred, B.Y., Kahn, A.E., et al., 2015. 
Controllability of structural brain networks. Nat. Commun. 6 .

Harriger, L., van den Heuvel, M.P., Sporns, O., 2012. Rich club organization of macaque cerebral cortex and its role in network communication. PloS One 7 (9), e46497.

Hiltunen, T., Kantola, J., Elseoud, A.A., Lepola, P., Suominen, K., Starck, T., Palva, J.M., 2014. Infra-slow EEG fluctuations are correlated with resting-state network dynamics in fMRI. J. Neurosci. 34 (2), 356-362. http://dx.doi.org/10.1523/ JNEUROSCI.0276-13.2014.

Hipp, J.F., Hawellek, D.J., Corbetta, M., Siegel, M., Engel, A.K., 2012. Large-scale cortical correlation structure of spontaneous oscillatory activity. Nat. Neurosci. 15 (6), 884-890.

Honey, C.J., Sporns, O., Cammoun, L., Gigandet, X., Thiran, J.P., Meuli, R., Hagmann, P., 2009. Predicting human resting-state functional connectivity from structural connectivity. Proc. Natl. Acad. Sci. USA 106 (6), 2035.

Hutchison, R.M., Womelsdorf, T., Allen, E.A., Bandettini, P.A., Calhoun, V.D., Corbetta, M., et al., 2013. Dynamic functional connectivity: promise, issues, and interpretations. Neuroimage 80, 360-378.

Kirchner, W.K., 1958. Age differences in short-term retention of rapidly changing information. J. Exp. Psychol. 55 (4), 352.

Lashley, K.S., 1929. Brain mechanisms and intelligence: A quantitative study of injuries to the brain.

Leech, R., Braga, R., Sharp, D.J., 2012. Echoes of the brain within the posterior cingulate cortex. J. Neurosci. 32 (1), 215-222. http://dx.doi.org/10.1523/JNEUROSCI.368911.2012 .

Liu, Y.-Y., Slotine, J.-J., Barabási, A.-L., 2011. Controllability of complex networks. Nature 473 (7346), 167-173. http://dx.doi.org/10.1038/nature10011.

Lőrincz, M.L., Geall, F., Bao, Y., Crunelli, V., Hughes, S.W., 2009. ATP-dependent infraslow $(<0.1 \mathrm{~Hz})$ oscillations in Thalamic networks. PLoS One 4 (2), e4447. http:// dx.doi.org/10.1371/journal.pone.0004447.

Maslov, S., Sneppen, K., 2002. Specificity and stability in topology of protein networks. Science 296 (5569), 910-913. http://dx.doi.org/10.1126/science.1065103.

Michalareas, G., Vezoli, J., van Pelt, S., Schoffelen, J.-M., Kennedy, H., Fries, P., 2016. Alpha-beta and gamma rhythms subserve feedback and feedforward influences among human visual cortical areas. Neuron.

Monto, S., Palva, S., Voipio, J., Palva, J.M., 2008. Very slow eeg fluctuations predict the dynamics of stimulus detection and oscillation amplitudes in humans. J. Neurosci. 28 (33), 8268-8272. http://dx.doi.org/10.1523/JNEUROSCI.1910-08.2008.

Moussa, M.N., Steen, M.R., Laurienti, P.J., Hayasaka, S., 2012. Consistency of network modules in resting-state fMRI connectome data. PLoS One 7 (8), e44428. http:// dx.doi.org/10.1371/journal.pone.0044428.

Patel, A.X., Bullmore, E.T., 2015. A wavelet-based estimator of the degrees of freedom in denoised fMRI time series for probabilistic testing of functional connectivity and brain graphs. NeuroImage.

Poldrack, R.A., Kittur, A., Kalar, D., Miller, E., Seppa, C., Gil, Y., Bilder, R.M., 2011. The cognitive atlas: toward a knowledge foundation for cognitive neuroscience. Front. Neuroinformatics $5(0)$

Reuter, M., Schmansky, N.J., Rosas, H.D., Fischl, B., 2012. Within-subject template estimation for unbiased longitudinal image analysis. Neuroimage 61 (4), $1402-1418$

Schmidt, R., LaFleur, K.J.R., de Reus, M.A., van den Berg, L.H., van den Heuvel, M.P., 2015. Kuramoto model simulation of neural hubs and dynamic synchrony in the human cerebral connectome. BMC Neurosci. 16 (1), 1.

Senden, M., Deco, G., de Reus, M.A., Goebel, R., van den Heuvel, M.P., 2014. Rich club organization supports a diverse set of functional network configurations. NeuroImage 96, 174-182. http://dx.doi.org/10.1016/j.neuroimage.2014.03.066.

Shanahan, M., 2012. The brain's connective core and its role in animal cognition. Philos Trans. R. Soc. Lond. B: Biol. Sci. 367 (1603), 2704-2714. http://dx.doi.org/ $10.1098 /$ rstb. 2012.0128.

Shepard, R.N., Metzler, J., 1971. Mental rotation of three-dimensional objects.

Smith, S.M., Fox, P.T., Miller, K.L., Glahn, D.C., Fox, P.M., Mackay, C.E., et al., 2009. Correspondence of the brain's functional architecture during activation and rest. Proc. Natl. Acad. Sci. USA 106 (31), 13040-13045.

Tononi, G., 2004. An information integration theory of consciousness. BMC Neurosci. 5 (1), 42.

van den Heuvel, M.P., Kahn, R.S., Goñi, J., Sporns, O., 2012. High-cost, high-capacity backbone for global brain communication. Proc. Natl. Acad. Sci. 109 (28), $11372-11377$.

van den Heuvel, M.P., de Reus, M.A., Feldman Barrett, L., Scholtens, L.H., Coopmans, F.M.T., Schmidt, R., Li, L., 2015. Comparison of diffusion tractography and tracttracing measures of connectivity strength in rhesus macaque connectome. Human. Brain Mapp. 36 (8), 3064-3075. http://dx.doi.org/10.1002/hbm.22828.

van den Heuvel, M.P., Sporns, O., 2011. Rich-club organization of the human connectome. J. Neurosci. 31 (44), 15775-15786.

Van Essen, D.C., Ugurbil, K., Auerbach, E., Barch, D., Behrens, T.E.J., Bucholz, R., et al., 2012. The Human Connectome Project: a data acquisition perspective. Neuroimage 62 (4), 2222-2231.

Vanhatalo, S., Palva, J.M., Holmes, M.D., Miller, J.W., Voipio, J., Kaila, K., 2004 Infraslow oscillations modulate excitability and interictal epileptic activity in the human cortex during sleep. Proc. Natl. Acad. Sci. USA 101 (14), 5053-5057.

Váša, F., Shanahan, M., Hellyer, P.J., Scott, G., Cabral, J., Leech, R., 2015. Effects of lesions on synchrony and metastability in cortical networks. NeuroImage 118 , 456-467. http://dx.doi.org/10.1016/j.neuroimage.2015.05.042.

Wildie, M., Shanahan, M., 2012. Metastability and chimera states in modular delay and pulse-coupled oscillator networks. Chaos: Interdiscip. J. Nonlinear Sci. 22 (4), 43131.

Yeh, F.-C., Wedeen, V.J., Tseng, W.-Y.I., 2010. Generalized-sampling imaging. IEEE Trans. Med. Imaging 29 (9), 1626-1635.

Zamora-López, G., Zhou, C., Kurths, J., 2009. Graph analysis of cortical networks reveals complex anatomical communication substrate. Chaos: Interdiscip. J. Nonlinear Sci. 19 (1), 15117.

Zhou, S., Mondragon, R.J., 2004. The rich-club phenomenon in the Internet topology. IEEE Commun. Lett. 8 (3), 180-182. http://dx.doi.org/10.1109/ LCOMM.2004.823426. 